\title{
On the range of the derivatives of a smooth function between Banach spaces $\dagger$
}

\author{
By D. AZAGRA, M. JIMÉNEZ-SEVILLA \\ Departamento de Análisis Matemático, Facultad de Ciencias Matemáticas, \\ Universidad Complutense, 28040 Madrid, Spain. \\ e-mail: Daniel_Azagra@mat.ucm.es, marjim@sunam1.mat.ucm.es \\ AND R. DEVILLE \\ Université Bordeaux I, 351, cours de la Libération, \\ 33045 Talence Cedex, France. \\ e-mail: deville@math.u-bordeaux.fr
}

\begin{abstract}
We study the size of the range of the derivatives of a smooth function between Banach spaces. We establish conditions on a pair of Banach spaces $X$ and $Y$ to ensure the existence of a $C^{p}$ smooth (Fréchet smooth or a continuous Gâteaux smooth) function $f$ from $X$ onto $Y$ such that $f$ vanishes outside a bounded set and all the derivatives of $f$ are surjections. In particular we deduce the following results. For the Gâteaux case, when $X$ and $Y$ are separable and $X$ is infinite-dimensional, there exists a continuous Gâteaux smooth function $f$ from $X$ to $Y$, with bounded support, so that $f^{\prime}(X)=\mathscr{L}(X, Y)$. In the Fréchet case, we get that if a Banach space $X$ has a Fréchet smooth bump and dens $X=$ dens $\mathscr{L}(X, Y)$, then there is a Fréchet smooth function $f: X \rightarrow Y$ with bounded support so that $f^{\prime}(X)=\mathscr{L}(X, Y)$. Moreover, we see that if $X$ has a $C^{p}$ smooth bump with bounded derivatives and dens $X=\operatorname{dens} \mathscr{L}_{s}^{m}(X ; Y)$ then there exists another $C^{p}$ smooth function $f: X \rightarrow Y$ so that $f^{(k)}(X)=\mathscr{L}_{s}^{k}(X ; Y)$ for all $k=0,1, \ldots, m$. As an application, we show that every bounded starlike body on a separable Banach space $X$ with a (Fréchet or Gâteaux) smooth bump can be uniformly approximated by smooth bounded starlike bodies whose cones of tangent hyperplanes fill the dual space $X^{*}$. In the non-separable case, we prove that $X$ has such property if $X$ has smooth partitions of unity.
\end{abstract}

\section{Introduction}

Although Rolle's theorem fails in infinite dimensions [2], Ekeland's Variational Principle [7, proposition II.5.2] easily determines that if $b$ is a continuous Gâteaux smooth bump on $X$ then the norm closure of $b^{\prime}(X)$ contains 0 as an interior point. If, in addition, $X$ is finite dimensional and $b$ is $C^{1}$ then $b^{\prime}(X)$ is a compact neighbourhood of 0 . The questions as to how small or how large the set of gradients of a bump

$\dagger$ Supported in part by DGICYT grant PB96-0607 and BFM 2000-0609. 
function can be have been considered recently in [1] and [3]. We refer to [5] for a study on the shape of the set $b^{\prime}(X)$ when $X$ is finite-dimensional.

On the one hand, as a consequence of a result of [11], we know that if $f$ is a $C^{1}$ smooth function defined on $c_{0}$ with locally uniformly continuous derivative then $f^{\prime}(X)$ is contained in a countable union of compacts. Also, if $X$ is a non-reflexive Banach space with a $C^{1}$ smooth norm, then the set of norm attaining functionals has empty interior in $X^{*}[\mathbf{1 2}]$ (see also [6]); by composing this norm with an adequate bump function on $\mathbb{R}$ one can easily obtain a $C^{1}$ smooth Lipschitz bump $b$ on $X$ so that the cone generated by $b^{\prime}(X)$, that is $C(b)=\left\{\lambda b^{\prime}(x): x \in X, \lambda \geqslant 0\right\}$, has empty interior.

If a continuous Gâteaux smooth bump $b$ is defined on a Banach space $X$ with the Radon Nikodym Property, it easily follows, as a consequence of Stegall's Variational Principle, that $C(b)$ is a residual set in $X^{*}$. Nevertheless, it may happen that $C(b)$ has empty interior even though $b$ is defined on a reflexive Banach space. Indeed, it has been proved in [3] that there exists a $C^{1}$ smooth Lipschitz bump $b$ defined on $\ell_{2}$ so that $C(b)$ has empty interior.

On the other hand, it was shown in [1] that there is no upper bound on the size of $b^{\prime}(X)$. More specifically, if $X$ has a $C^{1}$ smooth Lipschitz bump, then there is another $C^{1}$ smooth bump $b$ so that $b^{\prime}(X)=X^{*}$. This result can be compared to that of [4]: for every infinite-dimensional separable Banach spaces $X, Y$, there exists a $C^{1}$ smooth surjection from $X$ onto $Y$. T. Dobrowolski has recently obtained similar results for weak bump functions with higher order derivatives which are surjections (see [9]).

In this paper we improve the main results from [1], establishing several interesting generalizations which simultaneously include some of the results of [4] and [1], as well as an analogous result for the Fréchet and Gâteaux smooth case, and we provide some applications.

If $X$ and $Y$ are Banach spaces, let $\mathscr{L}(X, Y)$ stand for the Banach space of all bounded linear operators from $X$ to $Y$. We give conditions on a pair of Banach spaces $X$ and $Y$ to ensure the existence of a Fréchet or continuous Gâteaux smooth surjection $f: X \rightarrow Y$ such that $f$ vanishes outside a bounded set and $f^{\prime}(X)=\mathscr{L}(X, Y)$. In particular, when $X$ and $Y$ are separable and $X$ is infinite-dimensional, there exists a uniformly Gâteaux smooth function $f$ from $X$ to $Y$, with bounded support, so that $f^{\prime}(X)$ contains the unit ball of the Banach space $\mathscr{L}(X, Y)$. We obtain as a corollary that every separable Banach space $X$ has a uniformly Gâteaux smooth bump $b$ so that $b^{\prime}(X)$ contains the dual unit ball of $X^{*}$, and therefore, there is a continuous Gâteaux smooth bump $g$ so that $g^{\prime}(X)=X^{*}$. In the Fréchet smooth case, we obtain that if a Banach space $X$ has a Fréchet smooth bump and dens $X=\operatorname{dens} \mathscr{L}(X, Y)$, then there is a Fréchet smooth function $f: X \rightarrow Y$ with bounded support so that $f^{\prime}(X)=\mathscr{L}(X, Y)$. One corollary to this result is that if a Banach space $X$ has a Fréchet smooth bump, then $X$ has a Fréchet smooth bump $b$ so that $b^{\prime}(X)=X^{*}$. Another corollary states that for every separable infinite-dimensional Banach space $Y$ and every $n \in \mathbb{N}$, there is a Fréchet smooth function $f: \mathbb{R}^{n} \rightarrow Y$, with bounded support, so that $f^{\prime}\left(\mathbb{R}^{n}\right)=\mathscr{L}\left(\mathbb{R}^{n}, Y\right)$.

Next, we also provide conditions on a pair of Banach spaces $X$ and $Y$ which ensure the existence of a $C^{p}$ smooth surjection $f: X \rightarrow Y$ such that $f$ vanishes outside a bounded set and the derivatives of $f$ are all surjections. We prove that if $X$ has a $C^{p}$ smooth bump with bounded derivatives and dens $X=\operatorname{dens} \mathscr{L}_{s}^{m}(X ; Y)$ then there 
exists another $C^{p}$ smooth function $f: X \rightarrow Y$, with bounded derivatives, so that $f$ vanishes outside the unit ball of $X$ and $f^{(k)}(X)$ contains the unit ball of $\mathscr{L}_{s}^{k}(X ; Y)$ for all $k=0,1, \ldots, m$ (notice that this conclusion is in fact equivalent to the assumption on $X)$; in particular, this implies that there is also a $C^{p}$ smooth surjection $b: X \rightarrow Y$ so that $b^{(k)}(X)=\mathscr{L}_{s}^{k}(X ; Y)$ for all $k=0,1, \ldots, m$. Here, $1 \leqslant p \leqslant \infty, m \in \mathbb{N}, \mathscr{L}_{s}^{k}(X ; Y)$ stands for the space of $k$-linear symmetric mappings from $X$ into $Y$, and dens $X$ denotes the character of density of a Banach space $X$. Note in particular that for $m=0$ we identify $Y=\mathscr{L}_{s}^{0}(X, Y)$ and we obtain a $C^{p}$ smooth surjection $b$ from $X$ onto $Y$, thus recovering a result of [4]. For some classical spaces $X$ and $Y$, such as the $\ell_{p}, c_{0}, L_{p}$ and Orlicz spaces, we also study when the above conditions for the existence of smooth functions with surjective derivatives are fulfilled.

Finally, we give some applications of these results. In [1] it was shown that James' Theorem fails for starlike bodies in all separable Banach spaces; in particular it was seen that if $X$ is infinite-dimensional and has a separable dual then there is a $C^{1}$ smooth bounded starlike body whose cone of tangent hyperplanes fills the dual space $X^{*}$. Here we prove that in fact every bounded starlike body in such a space $X$ can be uniformly approximated by $C^{1}$ smooth starlike bodies whose cones of tangent hyperplanes fill the dual $X^{*}$. If $X$ is separable but $X^{*}$ is not, a similar statement holds replacing $C^{1}$ smoothness by Gâteaux smoothness. Moreover, a (non-separable) Banach space $X$ has this property provided $X$ has smooth partitions of unity. We also consider a similar question about smooth approximation of continuous functions by smooth functions whose derivatives are surjections, and we show that if a Banach space $X$ has smooth partitions of unity then every continuous function on $X$ can be uniformly approximated by smooth functions with surjective derivatives.

\section{The range of the gradients of a continuous Gâteaux (Fréchet) smooth function between Banach spaces}

Theorem 2.1. Let $X$ and $Y$ be separable Banach spaces, where $X$ is infinitedimensional. Then, there is a uniformly Gâteaux smooth Lipschitz function $b: X \rightarrow Y$ with bounded support so that $b(X)$ contains the unit ball of $Y$ and $b^{\prime}(X)$ contains the unit ball of $\mathscr{L}(X, Y)$.

Consequently, there is a continuous Gâteaux smooth function $g: X \rightarrow Y$ with bounded support so that $g$ and $g^{\prime}$ are surjections, that $i s, g(X)=Y$ and $g^{\prime}(X)=\mathscr{L}(X, Y)$.

A consequence of this theorem is that there is no upper bound for the range of the set of gradients of a continuous Gâteaux smooth bump on a separable Banach space. This is formally stated in the following corollary.

Corollary 2.2. Every separable Banach space $X$ has a uniformly Gâteaux smooth Lipschitz bump buch that $b^{\prime}(X)$ contains the dual unit ball $B_{X^{*}}$. Consequently, $X$ has a continuous Gâteaux smooth bump g so that $g^{\prime}(X)=X^{*}$.

The following result concerns Fréchet smooth functions. It was proved in [1] that if a Banach space has a $C^{1}$ smooth and Lipschitz bump, then the space has a $C^{1}$ smooth and Lipschitz bump satisfying that the set of gradients fills the dual unit ball. The proof of this result, as well as the proofs given in the next section for the $C^{p}$ smooth case, strongly rely on the existence of a smooth bump function with bounded derivatives. This requirement allows us to obtain smooth functions with continuous 
surjective derivatives. If one is not interested in the continuity of the first derivative, one can dispense with that assumption, obtaining similar results on the existence of Fréchet smooth bumps whose sets of gradients fill the dual unit ball. Notice that it is still an open problem whether every Banach space with a Fréchet smooth bump has a Fréchet smooth bump with bounded derivative as well.

Theorem 2.3. Let $X$ be a Banach space with a Fréchet smooth bump and $Y$ a Banach space so that dens $X=$ dens $\mathscr{L}(X, Y)$. Then, there exists a Fréchet smooth function $g: X \rightarrow Y$ so that $g$ has bounded support, $g^{\prime}(X)=\mathscr{L}(X, Y)$ and, when $X$ is infinite dimensional, also $g(X)=Y$.

Corollary 2.4. Let $X$ be a Banach space with a Fréchet smooth bump. Then, $X$ has a Fréchet smooth bump $b$ so that $b^{\prime}(X)=X^{*}$.

It is straightforward to verify that a uniformly Gâteaux smooth continuous function $f: X \rightarrow Y$, defined on a finite-dimensional Banach space $X$, satisfies that $f^{\prime}$ is continuous. Therefore $f^{\prime}(X)$ is a countable union of compact subsets. If, in addition, $Y$ is infinite dimensional, then $f^{\prime}(X)$ cannot contain the unit ball of the infinite dimensional Banach space $\mathscr{L}(X, Y)$. Thus, there is no uniformly Gâteaux smooth function $f$ from a finite dimensional Banach space $X$ into an infinite dimensional Banach space $Y$ so that $f^{\prime}(X)$ contains the unit ball of $\mathscr{L}(X, Y)$. However, we establish as a corollary of Theorem $2 \cdot 3$ that, under the above conditions on $X$ and $Y$, there exists a Fréchet smooth function with bounded support from $X$ into $Y$ and whose set of gradients fills the space $\mathscr{L}(X, Y)$. We will not mention in this section the case when $X$ and $Y$ are finite dimensional since in Section 3 we will prove the existence of $C^{1}$ smooth functions with bounded support from $X$ to $Y$ whose derivatives are surjections.

Corollary 2.5. Let $Y$ be an infinite dimensional and separable Banach space and $n \in \mathbb{N}$. Then, there is a Fréchet smooth and Lipschitz function $b: \mathbb{R}^{n} \rightarrow Y$ with bounded support such that $b^{\prime}\left(\mathbb{R}^{n}\right)$ contains the unit ball of the space $\mathscr{L}\left(\mathbb{R}^{n}, Y\right)$.

Consequently, there is a Fréchet smooth function $g: \mathbb{R}^{n} \rightarrow Y$ with bounded support so that $g^{\prime}\left(\mathbb{R}^{n}\right)=\mathscr{L}\left(\mathbb{R}^{n}, Y\right)$.

Let us begin with the proofs of the above results. In order to prove Theorems $2 \cdot 1$ and $2 \cdot 3$ we first present a lemma which provides the necessary tools to construct such functions.

Consider in $\mathscr{L}(X, Y)$ the topology $\tau$ of the pointwise convergence on $X$ and denote by $B_{\mathscr{L}(X, Y)}$ the unit ball of $\mathscr{L}(X, Y)$. Let us turn our attention to the topological space $\left(B_{\mathscr{L}(X, Y)}, \tau\right)$. When, for instance $Y=\mathbb{R}$, the space $\left(B_{\mathscr{L}(X, Y)}, \tau\right)$ is $\left(B_{X^{*}}, w^{*}\right)$, where $w^{*}$ denotes the weak* topology in the dual unit ball $B_{X^{*}}$. Assume that $X$ is separable and fix a dense sequence $\left(x_{n}\right)$ in the unit ball of $X$ and a decreasing sequence of positive numbers $\left(\varepsilon_{n}\right)$ converging to 0 . It is well known that a countable basis of neighborhoods of a point $T \in B_{\mathscr{L}(X, Y)}$ in the topological space $\left(B_{\mathscr{L}(X, Y)}, \tau\right)$ is the family $\left(U_{n}\right)$, where

$$
U_{n}=\left\{S \in B_{\mathscr{L}(X, Y)}:\left\|(T-S)\left(x_{i}\right)\right\| \leqslant \varepsilon_{n}, \quad i=1, \ldots, n\right\} .
$$

The sequence $\left(U_{n}\right)$ is a decreasing family of $\tau$-closed and convex subsets of $B_{\mathscr{L}(X, Y)}$. It is also known that the space $\left(B_{\mathscr{L}(X, Y)}, \tau\right)$ is separable whenever $X$ and $Y$ so are. 
Indeed, take a dense sequence $\left(y_{n}\right)$ in the unit ball of $Y$. Now, for every $n \in \mathbb{N}$ and $\lambda=$ $(\lambda(1), \ldots, \lambda(n)) \in \mathbb{N}^{n}$, we select an operator $T_{\lambda} \in B_{\mathscr{L}(X, Y)}$ so that $\left\|T_{\lambda}\left(x_{i}\right)-y_{\lambda(i)}\right\|<\varepsilon_{n}$ for $i=1, \ldots, n$ (if there exists). It is straightforward to verify that the set

$$
\left\{T_{\lambda}: \lambda \in \mathbb{N}^{n}\right\}_{n \in \mathbb{N}}
$$

is dense in $\left(B_{\mathscr{L}(X, Y)}, \tau\right)$. The properties of the separability of $\left(B_{\mathscr{L}(X, Y)}, \tau\right)$ and the existence in this space of a countable basis of neighbourhoods for every element, give us the following lemma.

Lemma 2.6. Let $X$ and $Y$ be separable Banach spaces and $\left(V_{n}\right)$ be a decreasing family of $\tau$-closed, convex and symmetric subsets of $2 B_{\mathscr{L}(X, Y)}$ which is a base of neighbourhoods of 0 in $\left(2 B_{\mathscr{L}(X, Y)}, \tau\right)$. Then, there is a sequence $\left(T_{m}\right) \subset 2 B_{\mathscr{L}(X, Y)}$, with the property that for any $T \in B_{\mathscr{L}(X, Y)}$,

$$
T=\tau \text {-sum } \sum_{k} T_{m_{k}}, \quad \text { for some subsequence }\left(T_{m_{k}}\right) .
$$

Moreover, the sums satisfy the stronger condition

$$
T-\sum_{i \leqslant k} T_{m_{i}} \in V_{k} \quad \text { and } \quad\left\|\sum_{i \leqslant k} T_{m_{i}}\right\| \leqslant 1, \quad k \in \mathbb{N} .
$$

If we denote

$$
\begin{aligned}
\Sigma= & \left\{\sigma=(\sigma(n)) \in \mathbb{N}^{\mathbb{N}}:\left(T_{\sigma(n)}\right) \text { satisfies }(2 \cdot 2) \text { for some } T \in B_{\mathscr{L}(X, Y)}\right\}, \\
& \text { and } \Sigma_{n}=\left\{\sigma_{n}:=(\sigma(1), \ldots, \sigma(n)): \sigma \in \Sigma\right\},
\end{aligned}
$$

then, an element $\sigma \in \mathbb{N}^{\mathbb{N}}$ satisfies that $\sigma \in \Sigma$ if and only if $\sigma_{n}=(\sigma(1), \ldots, \sigma(n)) \in \Sigma_{n}$ for every $n$.

Proof of Lemma 2.6. Let us consider a dense sequence $\left(S_{n}\right)$ in $\left(B_{\mathscr{L}(X, Y)}, \tau\right)$. Then, for any $T \in B_{\mathscr{L}(X, Y)}, T=\tau$ - $\lim S_{n_{k}}$, where $\left(S_{n_{k}}\right)$ is a subsequence of $\left(S_{n}\right)$. Moreover, we may assume that $T-S_{n_{k}} \in V_{k}$, for every $k$. Now, we can write $T$ as the $\tau$-sum $S_{n_{1}}+\sum_{k}\left(S_{n_{k+1}}-S_{n_{k}}\right)$. We relabel $\left(S_{n}\right)_{n} \cup\left(S_{n}-S_{m}\right)_{n>m}$ as $\left(T_{n}\right)$. Notice that the sequence $\left(T_{n}\right)$ is included in $2 B_{\mathscr{L}(X, Y)}$. Then, for every $T \in B_{\mathscr{L}(X, Y)}$ there is a subsequence $\left(T_{m_{k}}\right)$ satisfying

$$
T=\tau-\operatorname{sum} \sum_{k} T_{m_{k}} .
$$

Moreover, this sum satisfies the stronger condition

$$
T-\sum_{i \leqslant k} T_{m_{i}}=T-S_{n_{k}} \in V_{k} \quad \text { and } \quad\left\|\sum_{i \leqslant k} T_{m_{i}}\right\|=\left\|S_{n_{k}}\right\| \leqslant 1 .
$$

In order to prove the last assertion of the lemma, notice that whenever $\sigma \in \mathbb{N}^{\mathbb{N}}$ satisfies that $\sigma_{n} \in \Sigma_{n}$ for every $n$, then by $(2 \cdot 2)$ we deduce that

$$
\sum_{i=k+1}^{k+m} T_{\sigma(i)} \in 2 V_{k} \quad \text { and } \quad\left\|\sum_{i \leqslant k} T_{\sigma(i)}\right\| \leqslant 1 \quad \text { for any } k, \quad m \in \mathbb{N} .
$$


Consequently, $\sum_{i} T_{\sigma(i)}$ is $\tau$-convergent and it follows from the above that

$$
\sum_{i \geqslant k+1} T_{\sigma(i)} \in 2 V_{k}
$$

Thus, if $T:=\tau$-sum $\sum_{i} T_{\sigma(i)}$, this sum trivially satisfies (2.2) and then $\sigma \in \Sigma$. This concludes the proof of Lemma $2 \cdot 6$.

Proof of Theorem 2.1. We first construct uniformly Gâteaux smooth functions $f, h: X \rightarrow Y$ with bounded supports so that $f^{\prime}(X)$ contains the unit ball of $\mathscr{L}(X, Y)$ and $h(X)$ contains the unit ball of $Y$. Next, we define the required function $b$ as the sum of suitable translations of $f$ and $h$ with disjoint supports. Finally, we shall construct $g$ by slightly modifying $b$. The proof is divided into several steps as follows.

Step 1: Definition of $f$. Since $X$ is separable there exists an equivalent uniformly Gâteaux smooth norm $\|\cdot\|$ on $X\left[7\right.$, p. 68]. Consider a $C^{\infty}$ bump on $\mathbb{R}$ which we denote by $\theta$ so that $\theta(t)=1$ for $|t| \leqslant \frac{1}{2}, \theta(t)=0$ whenever $|t| \geqslant 1, \theta(\mathbb{R})=[0,1]$ and $\sup _{t \in \mathbb{R}}\left|\theta^{\prime}(t)\right| \leqslant 3$.

Now we define the following sequence of bumps $h_{n}: X \rightarrow \mathbb{R}$ as $h_{n}(x)=\theta\left(\|x\|^{\frac{1}{n}}\right)$, $x \in X$. The bumps $\left(h_{n}\right)$ are uniformly Gâteaux smooth and verify that $h_{n}(x)=1$ for $\|x\| \leqslant 1 / 2^{n}, h_{n}(x)=0$ for $\|x\| \geqslant 1$ and $h(X)=[0,1]$. Also, $h_{n}$ satisfies that $h_{n}^{\prime}(x)=$ $1 / n\|x\|^{-1+\frac{1}{n}}\|\cdot\|^{\prime}(x) \theta^{\prime}\left(\|x\|^{\frac{1}{n}}\right)$ if $x \neq 0$ and $h_{n}^{\prime}(0)=0$. Therefore

$$
\sup _{x \in X}\|x\|\left\|h_{n}^{\prime}(x)\right\| \leqslant \frac{3}{n} .
$$

The length of an element $\sigma \in \mathbb{N}^{n}, \sigma:=(\sigma(1), \ldots, \sigma(n))$, is $n$ and it is denoted by $|\sigma|$. Let us consider $\Sigma^{\prime}=\bigcup_{n} \Sigma_{n}$, where the family $\left(\Sigma_{n}\right)_{n}$ is the one obtained in the Lemma $2 \cdot 6$. Now, as in [1], we take a sequence of positive real numbers $\left(r_{n}\right)_{n}$, where $r_{n+1}=r_{n} / 2^{n+3}$, as well as a family of points $\left(x_{\sigma}\right)_{\sigma \in \Sigma^{\prime}}$ in $\frac{1}{2} B_{X}$ satisfying

(1) $B\left(x_{\sigma}, r_{|\sigma|}\right) \subset \frac{1}{2} B_{X}$,

(2) if $\sigma \neq \sigma^{\prime}$ have length $n$, then $\left\|x_{\sigma}-x_{\sigma^{\prime}}\right\|>3 r_{n}$,

(3) if $|\sigma|=n,\left|\sigma^{\prime}\right|=n+1$ and $\sigma<\sigma^{\prime}$ (that is to say, $\sigma_{n}^{\prime}=\sigma$ ), then $\left\|x_{\sigma}-x_{\sigma^{\prime}}\right\|=$ $r_{n} / 2^{n+1}=4 r_{n+1}$.

Therefore, for every $n \in \mathbb{N}$, the set

$$
\bigcup_{\sigma \in \Sigma_{n}} B\left(x_{\sigma}, r_{n}\right)
$$

is a disjoint union of closed balls inside the ball $\frac{1}{2} B_{X}$. Also, if $\sigma, \sigma^{\prime} \in \Sigma^{\prime}$ and $\sigma<\sigma^{\prime}$, then $B\left(x_{\sigma^{\prime}}, r_{\left|\sigma^{\prime}\right|}\right) \subset B\left(x_{\sigma}, r_{|\sigma|} / 2^{|\sigma|}\right)$. In this way, we have for every chain $\sigma_{1}<\sigma_{2}<$ $\cdots<\sigma_{n}<\cdots$, where $\sigma_{n} \in \Sigma_{n}$ for every $n$, a nested sequence of balls $B\left(x_{\sigma_{1}}, r_{1}\right) \supset$ $B\left(x_{\sigma_{2}}, r_{2}\right) \supset \cdots \supset B\left(x_{\sigma_{n}}, r_{n}\right) \supset \cdots$ whose intersection is a single point. Notice that, if $\left(T_{m}\right)$ is the sequence obtained in Lemma $2 \cdot 6$, then the associated series $\sum T_{\sigma_{n}(n)}$ is $\tau$-convergent.

Let us define for every $n$ the function $f_{n}: X \rightarrow Y$ as follows

$$
f_{n}(x)=\sum_{\sigma \in \Sigma_{n}}\left\langle T_{\sigma(n)}, x-x_{\sigma}\right\rangle h_{n}\left(r_{n}^{-1}\left(x-x_{\sigma}\right)\right) .
$$

The support of each $f_{n}$ is included in the disjoint union of closed balls which appears in $(2 \cdot 4)$. Thus, for $n$ and $x$ fixed, the series which defines $f_{n}(x)$ has at most one non-null summand, which is the same in a neighbourhood of $x$. This clearly implies 
that each $f_{n}$ is continuous and Gâteaux smooth. Also, if $\left\|x-x_{\lambda}\right\| \leqslant r_{n} / 2^{n}$ for some $\lambda \in \Sigma_{n}$, then $b_{n}(x)=\left\langle T_{\lambda(n)}, x-x_{\lambda}\right\rangle$. We define $f: X \rightarrow Y$

$$
f(x)=\sum_{n} f_{n}(x), \quad x \in X .
$$

Let us check that $f$ is well defined and continuous. Indeed, $\left|f_{n}(x)\right| \leqslant 2 r_{n}$ for every $x \in X$ so the series $\sum_{n} f_{n}$ converges uniformly to $f$ in $X$ and the continuity of $f$ follows.

Step 2: The function $f$ is Gâteaux smooth. In order to prove the Gâteaux smoothness of $f$ let us fix $x \in X$ and $v$ in the unit ball of $X$ and consider

$$
\begin{gathered}
\varphi:[-1,1] \longrightarrow Y, \quad \varphi(t)=f(x+t v), \\
\varphi_{n}:[-1,1] \longrightarrow Y, \quad \varphi_{n}(t)=f_{n}(x+t v) .
\end{gathered}
$$

Then, $\varphi(t)=\sum_{n} \varphi_{n}(t)$, for $|t| \leqslant 1$. In what follows we prove that the series of gradients $\sum_{n} \varphi_{n}^{\prime}(t)$ is uniformly convergent for $|t| \leqslant 1$. Indeed, if $y=x+t v=\lim _{n} x_{\sigma_{n}}$ for some $\sigma \in \mathbb{N}^{\mathbb{N}}$ with the property that $\sigma_{n}=(\sigma(1), \ldots, \sigma(n)) \in \Sigma_{n}$, for every $n$, then condition (3) above implies that, $\left\|y-x_{\sigma_{n}}\right\|<5 r_{n+1}=5 r_{n} / 2^{n+3}<r_{n} / 2^{n}$ and then $\left\|r_{n}^{-1}\left(y-x_{\sigma_{n}}\right)\right\|<1 / 2^{n}$. This implies that $h_{n}$ is linear in a neighbourhood of $y$ and then

$$
\sum_{n \geqslant 1} \varphi_{n}^{\prime}(t)=\sum_{n \geqslant 1} T_{\sigma(n)}(v) .
$$

Otherwise, there is a positive integer $k$ and $\gamma \in \Sigma_{k}$ so that

$$
\begin{aligned}
\sum_{n \geqslant 1} \varphi_{n}^{\prime}(t)=\sum_{n=1}^{k-1} T_{\gamma(n)}(v)+ & T_{\gamma(k)}(v) h_{k}\left(r_{k}^{-1}\left(x+t v-x_{\gamma}\right)\right) \\
& +T_{\gamma(k)}\left(x+t v-x_{\gamma}\right) r_{k}^{-1} h_{k}^{\prime}\left(r_{k}^{-1}\left(x+t v-x_{\gamma}\right)\right)(v) .
\end{aligned}
$$

Therefore for $N \in \mathbb{N}$ we deduce from inequality $(2 \cdot 3)$ that,

$$
\begin{gathered}
\left\|\sum_{n \geqslant N} \varphi_{n}^{\prime}(t)\right\|=\left\|\sum_{n \geqslant N} T_{\sigma(n)}(v)\right\|, \quad \text { in the case }(2 \cdot 5), \\
\left\|\sum_{n \geqslant N} \varphi_{n}^{\prime}(t)\right\| \leqslant\left\|\sum_{n=N}^{k-1} T_{\gamma(n)}(v)\right\|+\left\|T_{\gamma(k)}(v)\right\|+\frac{6}{N}, \quad \text { in the case }(2 \cdot 6),
\end{gathered}
$$

(assuming that $k \geqslant N$, and 0 otherwise).

Inequalities (2.7) and (2.8) imply that $\sum_{n \geqslant N} \varphi_{n}^{\prime}(t)$ tends to 0 as $N$ tends to infinity uniformly on $[-1,1]$. Thus $\varphi(t)$ is differentiable and $\varphi^{\prime}(t)=\sum_{n} \varphi_{n}^{\prime}(t)$. In particular, $\varphi^{\prime}(0)=\sum_{n} f_{n}^{\prime}(x)(v)$. Since this can be done for every $v$ in the unit sphere of $X$, we deduce that $f$ is Gâteaux smooth at $x$ and

$$
\begin{gathered}
f^{\prime}(x)=\sum_{n} T_{\sigma(n)}, \quad \text { in the case }(2 \cdot 5) \\
f^{\prime}(x)=\sum_{n=1}^{k-1} T_{\gamma(n)}+T_{\gamma(k)} h_{k}\left(r_{k}^{-1}\left(x-x_{\gamma_{k}}\right)\right) \\
+T_{\gamma(k)}\left(x-x_{\gamma_{k}}\right) r_{k}^{-1} h_{k}^{\prime}\left(r_{k}^{-1}\left(x-x_{\gamma_{k}}\right)\right), \quad \text { in the case }(2 \cdot 6) .
\end{gathered}
$$


Notice that $f^{\prime}$ is bounded, thus $f$ is Lipschitz. The derivative $f^{\prime}$ is $\|\cdot\|-\tau$ continuous as well.

Step 3: The function $f$ is uniformly Gâteaux smooth. Fix $v$ in the unit sphere of $X$. Consider for every $n$, the function $G_{n}$, defined as

$$
G_{n}: X \longrightarrow Y, \quad G_{n}(x)=f_{n}^{\prime}(x)(v) .
$$

On the one hand, it is straightforward to verify that the function $G_{n}$ is uniformly continuous (this is a consequence of the uniformly Gâteaux smoothness of the norm $\|\cdot\|)$. On the other hand, it can be deduced from inequalities $(2 \cdot 7),(2 \cdot 8)$ and the strong property $(2 \cdot 2)$ related to the directionally uniform convergence of the series $\sum_{n} T_{\sigma(n)}$, that the series $\sum_{n} G_{n}$ is uniformly convergent on $X$. In particular, this implies that the limit function of the series $G: X \rightarrow Y, G(x)=\sum_{n} G_{n}(x)=f^{\prime}(x)(v)$ is uniformly continuous on $X$. If $v$ ranges over the norm one elements of $X$, we obtain the conclusion. In addition, we have that

$$
B_{\mathscr{L}(X, Y)}=\left\{\sum_{i} T_{\sigma(i)}: \sigma \in \Sigma\right\} \subset f^{\prime}(X) .
$$

Step 4: Construction of the functions $h$ and $b$. Following a similar construction, we obtain a uniformly Gâteaux smooth function $h$ with bounded support so that the image of $h$ fills the unit ball of $Y$ (see [4]). Let us give an outline of this assertion. Consider a dense sequence $\left\{y_{n}\right\}_{n}$ in the unit ball of $Y$. Fix $0<\varepsilon<\frac{1}{12}$ and select a family $\mathscr{F}=\left\{x_{\sigma}: \sigma \in \mathbb{N}^{n}, n \in \mathbb{N}\right\}$ in $\frac{1}{2} B_{X}$ satisfying the following conditions

(1) $B\left(x_{\sigma}, \varepsilon^{|\sigma|}\right) \subset \frac{1}{2} B_{X}$,

(2) if $\sigma \neq \sigma^{\prime}$ have length $n$, then $\left\|x_{\sigma}-x_{\sigma^{\prime}}\right\|>3 \varepsilon^{n}$,

(3) if $|\sigma|=n,\left|\sigma^{\prime}\right|=n+1$ and $\sigma<\sigma^{\prime}$ (that is to say, $\sigma_{n}^{\prime}=\sigma$ ), then $\left\|x_{\sigma}-x_{\sigma^{\prime}}\right\|=\varepsilon^{n} / 4$.

Then, for every $n$ and $x \in X$, we define $g_{n}: X \rightarrow Y$

$$
g_{n}(x)=\sum_{\sigma \in \mathbb{N}^{n}} \varepsilon^{2(n-1)} y_{\sigma(n)} h_{1}\left(\varepsilon^{-n}\left(x-x_{\sigma}\right)\right),
$$

and $h: X \rightarrow Y, h(x)=\sum_{n} g_{n}(x)$. The function $h$ is uniformly Gâteaux smooth, has bounded support and $h(X)$ contains the unit ball of $Y$ : for every $y \in B_{Y}$ there is $\sigma \in \mathbb{N}^{\mathbb{N}}$ so that $y=\sum \varepsilon^{2(n-1)} y_{\sigma(n)}$ and then, if $x=\lim _{n} x_{\sigma_{n}}$, we have that $h(x)=y$.

Now $b$ can be defined as $b(x)=f(x-a)+h(x+a)$, where $\|a\|=\frac{1}{2}$ and then $\operatorname{supp}(b) \subset B_{X}$. Obviously, from the definition and the fact that the supports of the above translations of $f$ and $h$ are disjoint, it follows that $b(X)$ contains the unit ball of $Y$ and $b^{\prime}(X)$ fills the unit ball of $\mathscr{L}(X, Y)$.

Step 5: The construction of the function $g$. In order to obtain a continuous and Gâteaux smooth function $g: X \rightarrow Y$ whose image fills $Y$ and whose set of gradients fills $\mathscr{L}(X, Y)$ we proceed in a standard way as follows: consider the above function $b$ whose support is included in $B_{X}$ and fix a sequence $\left(y_{n}\right)$ in the unit sphere of $X$ such that $\left\|y_{n}-y_{m}\right\| \geqslant \frac{1}{2}$ whenever $n \neq m$. Then we may define $g$ on $X$ as $g(x)=$ $\sum_{n} n b\left(4\left(x-y_{n}\right)\right)$. This concludes the proof of Theorem $2 \cdot 1$.

Proof of Theorem 2.3. Let us consider a Fréchet smooth bump $h: X \rightarrow \mathbb{R}$ so that $h(x)=1$ for $\|x\| \leqslant \delta$ (for some $0<\delta<\frac{1}{2}$ ), $h(x)=0$ whenever $\|x\| \geqslant 1$ and $h(X) \subset$ $[0,1]$. Let us define $r_{n}=\delta^{n-1} / 2^{n-1}[(n+2) !]^{2}$ for every $n \in \mathbb{N}$.

If $X$ is finite dimensional, we consider in $\left(B_{\mathscr{L}(X, Y)},\|\cdot\|\right)$ a dense set $\left\{T_{m}\right\}$, where 
$T_{1}=0$, and a decreasing sequence of positive numbers $\left\{\varepsilon_{n}\right\}$ converging to 0 . Then for every $T \in B_{\mathscr{L}(X, Y)}$,

$$
\begin{aligned}
& \quad T=\sum_{k} T_{\sigma(k)} \quad \text { for some } \sigma \in \mathbb{N}^{\mathbb{N}}, \quad \text { where } \sigma(k) \in\{1, k\}, \\
& \left\|T-\sum_{i \leqslant m_{k}} T_{\sigma(i)}\right\| \leqslant \varepsilon_{k}, \quad \text { for some } m_{1}<m_{2}<\cdots \text { and } \sigma(i)=1 \\
& \quad \text { if } \quad i \notin\left\{m_{1}, m_{2}, \ldots\right\} .
\end{aligned}
$$

If $X$ is infinite dimensional and dens $X=\kappa$, we consider in $\left(B_{\mathscr{L}(X, Y)},\|\cdot\|\right)$ a dense set $\left\{T_{\lambda}\right\}_{\lambda \in \kappa}$. Then, for every $T \in B_{\mathscr{L}(X, Y)}$ there is $\sigma \in \kappa^{\mathbb{N}}$ so that

$$
\left\|T-\sum_{i \leqslant k} T_{\sigma(i)}\right\| \leqslant \varepsilon_{k}, \quad \text { for every } k \in \mathbb{N} .
$$

If $X$ is finite dimensional, let us define

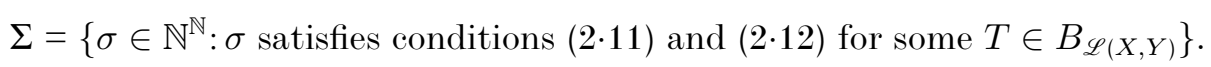

If $X$ is infinite dimensional, we define $\Sigma$ as

$$
\Sigma=\left\{\sigma \in \kappa^{\mathbb{N}}: \sigma \text { satisfies condition (2.13) for some } T \in B_{\mathscr{L}(X, Y)}\right\} \text {. }
$$

Denote $\Sigma_{n}=\left\{\sigma_{n}:=(\sigma(1), \ldots, \sigma(n)): \sigma \in \Sigma\right\}$ and $\Sigma^{\prime}=\bigcup_{n} \Sigma_{n}$. Then, it is straightforward to verify that, in both cases, $\sum_{k} T_{\sigma(k)}$ is convergent whenever $\sigma$ satisfies that $\sigma_{n} \in \Sigma_{n}$ for every $n \in \mathbb{N}$. Thus $\sigma \in \Sigma$ if and only if $\sigma_{n} \in \Sigma_{n}$ for every $n$.

Now, as in the preceding proof, let us take a family $\left(x_{\sigma}\right)_{\sigma \in \Sigma^{\prime}}$ in $\delta B_{X}$ so that

(1) $B\left(x_{\sigma}, r_{|\sigma|}\right) \subset \delta B_{X}$,

(2) if $\sigma \neq \sigma^{\prime}$ have length $n$, then $\left\|x_{\sigma}-x_{\sigma^{\prime}}\right\| \geqslant r_{n}(n+2)$,

(3) if $|\sigma|=n,\left|\sigma^{\prime}\right|=n+1$ and $\sigma<\sigma^{\prime}$ (that is to say, $\sigma_{n}^{\prime}=\sigma$ ), then $\left\|x_{\sigma}-x_{\sigma^{\prime}}\right\|=$ $\delta\left(r_{n} / n+2\right)$.

Therefore, for every $n \in \mathbb{N}$, the set

$$
\bigcup_{\sigma \in \Sigma_{n}} B\left(x_{\sigma}, r_{n}\right)
$$

is a disjoint union of closed balls inside the ball $\delta B_{X}$. Also, if $\sigma, \sigma^{\prime} \in \Sigma^{\prime}$ and $\sigma<\sigma^{\prime}$, then $B\left(x_{\sigma^{\prime}}, r_{\left|\sigma^{\prime}\right|}\right) \subset B\left(x_{\sigma}, 2 \delta r_{|\sigma|} /(|\sigma|+2)\right)$. When $X$ is finite dimensional, the disjoint union which appears in $(2 \cdot 14)$ consist at most of $2^{n-1}$ balls and every ball of radius $r_{n}$ contains at most a pair of balls of radius $r_{n+1}$.

First, let us define a Frêchet smooth function $b: X \rightarrow Y$ so that $b^{\prime}(X)$ contains the unit ball of $\mathscr{L}(X, Y)$ as the series $b(x)=\sum_{n} b_{n}(x)$, for $x \in X$, where the functions $b_{n}: X \rightarrow Y$ are defined by

$$
b_{n}(x)=\sum_{\sigma \in \Sigma_{n}}\left\langle T_{\sigma(n)}, x-x_{\sigma}\right\rangle h\left(r_{n}^{-1}\left(x-x_{\sigma}\right)\right) .
$$

Notice that the support of each $b_{n}$ is included in the disjoint union of closed balls which appears in $(2 \cdot 4)$. Thus, for $n$ and $x$ fixed, the series which defines $b_{n}(x)$ has at most one non-null summand, which is the same in a neighbourhood of $x$. This implies 
that each $b_{n}$ is continuous and Fréchet smooth. Now, $\left\|b_{n}(x)\right\| \leqslant r_{n}$ for every $x \in X$ so the series $\sum_{n} b_{n}$ converges uniformly to $b$ in $X$ and the continuity of $b$ follows.

In order to prove the Fréchet smoothness of $b$ let us take $x \in X$ so that there is $\sigma \in$ $\kappa^{\mathbb{N}}(\kappa=\operatorname{dens} X)$ with the property that $x=\lim _{n} x_{\sigma_{n}}$, where $\sigma_{n}=(\sigma(1), \ldots, \sigma(n)) \in$ $\Sigma_{n}$, for every $n$. Then by condition (3) above, $\left\|r_{n}^{-1}\left(x-x_{\sigma_{n}}\right)\right\|<\delta /(n+2)<\delta$ and

$$
b(x)=\sum_{n}\left\langle T_{\sigma(n)}, x-x_{\sigma_{n}}\right\rangle .
$$

Now, if $t>0,\|v\|=1$ and $x+t v \in B\left(x_{\sigma_{n}}, r_{n}\right) \backslash B\left(x_{\sigma_{n+1}}, r_{n+1}\right)$, there exists $\sigma^{\prime} \in \kappa^{\mathbb{N}}$ so that $\sigma_{n}^{\prime}=\sigma_{n}$ and

$$
\begin{aligned}
b(x+t v)=\sum_{i=1}^{n-1}\left\langle T_{\sigma(i)} x+t v\right. & \left.-x_{\sigma_{i}}\right\rangle+\left\langle T_{\sigma(n)}, x+t v-x_{\sigma_{n}}\right\rangle h\left(r_{n}^{-1}\left(x+t v-x_{\sigma_{n}}\right)\right) \\
+ & \sum_{i \geqslant n+1}\left\langle T_{\sigma^{\prime}(i)}, x+t v-x_{\sigma_{i}^{\prime}}\right\rangle h\left(r_{i}^{-1}\left(x+t v-x_{\sigma_{i}^{\prime}}\right)\right),
\end{aligned}
$$

where the last summand may be a eventually null series. Then,

$$
\begin{aligned}
& \left\|\frac{b(x+t v)-b(x)}{t}-\left\langle\sum_{i \geqslant 1} T_{\sigma(i)}, v\right\rangle\right\| \\
& \leqslant\left\|\left\langle\sum_{i \geqslant n} T_{\sigma(i)}, v\right\rangle\right\|+\left\|\left\langle T_{\sigma(n)}, v\right\rangle\right\|\left|h\left(r_{n}^{-1}\left(x+t v-x_{\sigma_{n}}\right)\right)\right| \\
& \quad+\frac{1}{t}\left\|\left\langle T_{\sigma(n)}, x-x_{\sigma_{n}}\right\rangle\right\|\left|h\left(r_{n}^{-1}\left(x+t v-x_{\sigma_{n}}\right)\right)-1\right| \\
& \quad+\frac{1}{t} \sum_{i \geqslant n+1}\left\|\left\langle T_{\sigma^{\prime}(i)}, x+t v-x_{\sigma_{i}^{\prime}}\right\rangle\right\|\left|h\left(r_{i}^{-1}\left(x+t v-x_{\sigma_{i}^{\prime}}\right)\right)\right| \\
& \quad+\frac{1}{t} \sum_{i \geqslant n+1}\left\|\left\langle T_{\sigma(i)}, x-x_{\sigma_{i}}\right\rangle\right\| .
\end{aligned}
$$

The first and second summand in the second member of the above inequality may be arbitrarily small for $n$ big enough because $\sum_{i \geqslant n} T_{\sigma(i)}$ is convergent. In the case of the third summand notice that if $h\left(r_{n}^{-1}\left(x+t v-x_{\sigma_{n}}\right)\right) \neq 1$ then $t \geqslant \delta r_{n}-\left\|x-x_{\sigma_{n}}\right\| \geqslant$ $\delta r_{n}-\delta r_{n} /(n+2) \geqslant \frac{2}{3} \delta r_{n}$ and then

$$
\frac{1}{t}\left\|\left\langle T_{\sigma(n)}, x-x_{\sigma_{n}}\right\rangle\right\| \mid h\left(r_{n}^{-1}\left(x+t v-x_{\sigma_{n}}\right)\right)-1\left\|\leqslant \frac{1}{t}\right\| x-x_{\sigma_{n}} \| \leqslant \frac{3}{2(n+2)} .
$$

Now, if the fourth summand is not null then there exists $k \geqslant n+1$ so that $h\left(r_{k}^{-1}\left(x+t v-x_{\sigma_{k}^{\prime}}\right)\right) \neq 0$ and then $h\left(r_{n+1}^{-1}\left(x+t v-x_{\sigma_{n+1}^{\prime}}\right)\right) \neq 0$ and $x+t v \in B\left(x_{\sigma_{n+1}^{\prime}}, r_{n+1}\right)$. Since $\sigma_{n+1}^{\prime} \neq \sigma_{n+1}$ we have that $t \geqslant r_{n+1}(n+3)-2 r_{n+1}=r_{n+1}(n+1)$. Therefore we have the inequality

$$
\begin{aligned}
& \frac{1}{t} \sum_{i \geqslant n+1}\left\|\left\langle T_{\sigma^{\prime}(i)}, x+t v-x_{\sigma_{i}^{\prime}}\right\rangle\right\|\left|h\left(r_{i}^{-1}\left(x+t v-x_{\sigma_{i}^{\prime}}\right)\right)\right| \\
& \quad \leqslant \frac{1}{n+1} \sum_{i \geqslant n+1} r_{i} r_{n+1}^{-1}<\frac{2}{(2-\delta)(n+1)} .
\end{aligned}
$$


Finally,

$$
\begin{aligned}
\frac{1}{t} \sum_{i \geqslant n+1}\left\|\left\langle T_{\sigma(i)}, x-x_{\sigma_{i}}\right\rangle\right\| & \leqslant \frac{\sum_{i \geqslant n+1}\left(\delta r_{i} / i+2\right)}{r_{n+1}-\left(2 \delta r_{n+1}\right) /(n+3)} \\
& \leqslant \frac{2}{2-\delta} \sum_{i \geqslant n+1} \frac{r_{n+1}^{-1} r_{i}}{i+2} \leqslant \frac{4}{(n+3)(2-\delta)^{2}} .
\end{aligned}
$$

This proves that $b$ is Fréchet smooth at $x=\lim _{n} x_{\sigma_{n}}$ with $b^{\prime}(x)=\sum_{n} T_{\sigma(n)}$.

The Fréchet smoothness at the rest of the points follows immediately since $b$ can be written as a finite sum of the terms which appear in the series which defines $b$ in a neighbourhood of each one of these points. In this case $b^{\prime}(x)=\sum_{1}^{N-1} T_{\sigma(i)}+$ $T_{\sigma(N)} h\left(r_{N}^{-1}\left(x-x_{\sigma(N)}\right)\right)+\left\langle T_{\sigma(N)}, x-x_{\sigma_{N}}\right\rangle r_{N}^{-1} h^{\prime}\left(r_{N}^{-1}\left(x-x_{\sigma_{N}}\right)\right)$, for some $N$ and $\sigma_{N} \in \Sigma_{N}$.

From the above we deduce that $b^{\prime}$ is bounded in $X$ whenever $h^{\prime}$ is bounded in $X$. This is the case for instance when $X$ is separable and, in particular, when $X$ is finite dimensional as in Corollary $2 \cdot 5$.

Finally, $b$ satisfies the required conditions since

$$
B_{\mathscr{L}(X, Y)}=\left\{\sum_{i} T_{\sigma(i)}: \sigma \in \Sigma\right\} \subset b^{\prime}(X) .
$$

In order to obtain a $g$ such that $g^{\prime}$ is surjective, and when $X$ is infinite dimensional, also $g$ is surjective, we proceed in a standard way as in the above proof.

\section{Higher order derivatives which are surjections}

In this section we deal with the following question. When can one construct a $C^{p}$ smooth mapping $f$ between two Banach spaces $X$ and $Y$ such that $f$ has a bounded support and the derivatives $f^{(k)}, k=0,1, \ldots, p$, are all surjections (i.e. $f(X)=Y$ and $f^{(k)}(X)=\mathscr{L}_{s}^{k}(X ; Y)$ for all $k=1, \ldots, p$, where $\mathscr{L}_{s}^{k}(X ; Y)$ is the space of $k$-linear symmetric and continuous mappings from $X$ into $Y)$ ?

To begin with, it should be noted that, even in the simplest case when $Y=\mathbb{R}$, there are very smooth separable Banach spaces $X$ for which this is not possible at all, since the spaces $\mathscr{L}_{s}^{k}(X)$ need not be separable for $k \geqslant 2$ (here we denote $\mathscr{L}_{s}^{k}(X ; \mathbb{R})=\mathscr{L}_{s}^{k}(X)$, the space of $k$-linear symmetric and continuous forms on $X$, which is isomorphic to $\mathscr{P}^{(k)}(X)$, the space of $k$-homogeneous and continuous polynomials on $X)$. For instance, if $X=\ell_{2}$ then no $C^{2}$ smooth bump $b$ on $X$ has the property that $b^{2}(X)=\mathscr{L}_{s}^{2}(X)$; indeed, since $b^{(2)}$ is continuous and $X$ is separable, $b^{(2)}(X)$ is separable as well and hence cannot fill all of $\mathscr{L}_{s}^{2}(X)$, which is non-separable (to see this, notice that the mapping $a=\left(a_{n}\right) \mapsto A(x, y)=\sum_{n=1}^{\infty} a_{n} x_{n} y_{n}$ defines an isometric embedding of $\ell_{\infty}$ into the space of bilinear forms on $X=\ell_{2}$ ). More generally, it is known that if $X=\ell_{p}$ then the spaces $\mathscr{L}_{s}^{k}(X)$ are separable if and only if $k<[p]$, where $[p]$ is the integer part of $p$ (see $[8]$ for instance).

The above argument clearly shows that dens $X=\operatorname{dens} \mathscr{L}_{s}^{m}(X ; Y)$ is a necessary condition for a pair of Banach spaces $X$ and $Y$ to have a $C^{p}$ smooth function $f$ from $X$ onto $Y$ so that $f^{(k)}(X)=\mathscr{L}_{s}^{k}(X)$ for all $k=0,1, \ldots, m$. The next result (which can be regarded as a generalization of both one of the main theorems in $[1]$ and another in [4]) tells us that if the Banach space $X$ has a $C^{p}$ smooth bump with bounded 
derivatives then this condition is sufficient as well. In the following statement we use the convention $\mathscr{L}_{s}^{0}(X ; Y)=Y$ and $f^{(0)}=f$.

Theorem 3.1. Let $m, p \in\{0,1,2, \ldots, \infty\}$ and let $X, Y$ be Banach spaces with $\operatorname{dim} X=\infty$. The following are equivalent:

(1) $X$ has a $C^{p}$ smooth bump function with bounded derivatives, and $\operatorname{dens} X=$ dens $\mathscr{L}_{s}^{m}(X ; Y)$;

(2) there is a $C^{p}$ smooth function $f: X \rightarrow Y$, with bounded derivatives and bounded support, such that $f^{(k)}(X)$ contains the unit ball of $\mathscr{L}_{s}^{k}(X ; Y)$ for every $k=$ $0,1, \ldots, m$.

In particular, if $X$ satisfies condition (1) then there is another $C^{p}$ smooth function $b$ from $X$ onto $Y$ with bounded support so that its derivatives are all surjections up to the degree $m$, that is, $b^{(k)}(X)=\mathscr{L}_{s}^{k}(X ; Y)$, for $k=0,1, \ldots, m$.

Before proceeding with the proof of this result let us state some remarkable consequences. First, notice that when $m=0$ and dens $X=\operatorname{dens} Y$ we recover a particular case of a result of $[4]$.

Corollary 3.2. Let $X$ and $Y$ be Banach spaces with $\operatorname{dens} X \geqslant \operatorname{dens} Y, \operatorname{dim} X=\infty$, and assume that $X$ has a $C^{p}$ smooth bump function with bounded derivatives ( $p=$ $1,2, \ldots, \infty)$. Then there is a $C^{p}$ smooth surjection $f: X \rightarrow Y$ whose support is in the unit ball of $X$; moreover, if we additionally assume that dens $X=\operatorname{dens} \mathscr{L}(X, Y)$, the derivative $f^{\prime}$ is a continuous surjection as well, that is, $f^{\prime}(X)=\mathscr{L}(X, Y)$.

When $m=1$ and $Y=\mathbb{R}$ Theorem $3 \cdot 1$ yields the following improvement of one of the main results in $[\mathbf{1}]$.

Corollary 3י3. Let $X$ be an infinite-dimensional Banach space and $p \in\{1,2, \ldots, \infty\}$. The following are equivalent:

(1) $X$ has a $C^{p}$ smooth bump function with bounded derivatives;

(2) $X$ has a $C^{p}$ smooth bump function $f$, with bounded derivatives, so that $f^{\prime}(X)$ contains the unit ball of $X^{*}$.

In either case, there exists another $C^{p}$ smooth bump $b$ on $X$ so that $b^{\prime}(X)=X^{*}$.

It should be noted that if a Banach space $X$ satisfies condition (1) of Theorem $3 \cdot 1$ for $p \geqslant 2$ then it is super-reflexive (see [7]). Let us mention that condition dens $X=$ dens $\mathscr{L}_{s}^{k}(X, Y)$ is strongly related to Gonzalo and Jaramillo indexes $\ell(X), \ell(Y)$ and $u(X), u(Y)$ concerning upper and lower estimates of the Banach spaces $X$ and $Y$ (see [8] and [10]). For instance, it is proved in [8] that if a Banach space has an unconditional and shrinking basis then $\mathscr{L}_{s}^{k}(X)$ is separable if and only if every $T \in$ $\mathscr{L}_{s}^{k}(X)$ is weakly sequentially continuous which is equivalent to the fact that the Banach space $\mathscr{L}_{s}^{k}(X)$ has a monomial basis. Also, it is proved that (a) if $X$ has a shrinking basis and $(k-1) u\left(X^{*}\right)<\ell(X)$, then $\mathscr{L}_{s}^{k}(X)$ has a monomial basis (and thus it is separable); (b) if $X$ has an unconditional and shrinking basis and $u(X)<k$, then $\mathscr{L}_{s}^{k}(X)$ contains $\ell_{\infty}$.

If we apply Theorem $3 \cdot 1$ to some of the classic Banach spaces, we get the following corollaries. For a definition of the indexes $\alpha_{M}$ and $\alpha_{M}^{\infty}$ in Orlicz spaces and the best order of smoothness of these spaces (see [13]). 
Corollary $3 \cdot 4$.

(1) $c_{0}$ has $a C^{n}$ smooth bump $b$ with $b^{\prime}\left(c_{0}\right)=\ell_{1}$ if and only if $n=1$.

(2) For $r$ an even integer, the space $\ell_{r}$ has a $C^{\infty}$ smooth bump b with $b^{(k)}\left(\ell_{r}\right)=\mathscr{L}_{s}^{k}\left(\ell_{r}\right)$ for $k=1,2, \ldots, m$ if and only if $m<r$.

(3) If $r$ is not an even integer, $\ell_{r}$ has a $C^{m}$ smooth bump b with $b^{(k)}\left(\ell_{r}\right)=\mathscr{L}_{s}^{k}\left(\ell_{r}\right)$ for $k=1, \ldots, m$ if and only if $m<r$.

(4) If $\ell_{M}$ is a reflexive Orlicz sequence space and $\alpha_{M}$ is the lower index of Boyd, then for $m<\alpha_{M}, \ell_{M}$ has a $C^{m}$ smooth bump b so that $b^{(k)}\left(\ell_{M}\right)=\mathscr{L}_{s}^{k}\left(\ell_{M}\right)$, whenever $k \in\{1, \ldots, m\}$.

Notice that according to a result of [11], no $C^{2}$-smooth bump $b$ on $c_{0}$ has the property that $b^{\prime}\left(c_{0}\right)=\ell_{1}$ and assertion (1) in the above corollary follows.

The classical Banach space $L_{r}[0,1], r \geqslant 1$, as well as the Lorentz function spaces $L_{M}[0,1]$ contain a complemented copy of $\ell_{2}$. Thus $\mathscr{L}_{s}^{2}\left(L_{r}[0,1]\right)$ and $\mathscr{L}_{s}^{2}\left(L_{M}[0,1]\right)$ contain $\ell_{\infty}$ and the best we can expect for these spaces is the following result.

Corollary $\mathbf{3} \cdot \mathbf{5}$.

(1) For $r$ an even integer the space $L_{r}[0,1]$ has a $C^{\infty}$ smooth bump so that $b^{\prime}\left(L_{r}[0,1]\right)=$ $L_{r^{\prime}}[0,1], 1 / r+1 / r^{\prime}=1$.

(2) If $r$ is not an even integer, the space $L_{r}[0,1]$ has a $C^{m}$ smooth bump $b$ so that $b^{\prime}\left(L_{r}[0,1]\right)=L_{r^{\prime}}[0,1]$ if and only if $m<r$.

(3) If $L_{M}[0,1]$ is a reflexive Orlicz function space and $\alpha_{M}^{\infty}$ is the lower index of Boyd at $\infty$, then for $m<\alpha_{M}^{\infty}, L_{M}[0,1]$ has a $C^{m}$ smooth bump bo that $b^{\prime}\left(L_{M}[0,1]\right)=$ $\left.\mathscr{L}_{M^{*}}[0,1]\right)$, where $M^{*}$ is the Young conjugate of $M$.

In the vector valued case let us mention that $\mathscr{L}\left(c_{0}, \ell_{1}\right)$ is separable, and $\mathscr{L}_{s}^{k}\left(\ell_{r}, \ell_{q}\right)$ is separable if and only if $k q<r$. Thus we obtain for these spaces the following result.

Corollary $3 \cdot 6$.

(1) There is a $C^{1}$ smooth function $f: c_{0} \rightarrow \ell_{1}$ with bounded support so that $f\left(c_{0}\right)=\ell_{1}$ and $f^{\prime}\left(c_{0}\right)=\mathscr{L}\left(c_{0} ; \ell_{1}\right)$.

(2) When $m q<r$, there is a $C^{m}$ smooth function $f: \ell_{r} \rightarrow \ell_{q}$ with bounded support so that $f^{(k)}\left(\ell_{r}\right)=\mathscr{L}_{s}^{k}\left(\ell_{r} ; \ell_{q}\right)$ for $k \in\{0,1, \ldots, m\}$.

What about Theorem $3 \cdot 1$ when $X$ and $Y$ are finite-dimensional? In this case, an analogous result is available which provides us with Peano functions from $\mathbb{R}^{k}$ to $\mathbb{R}^{m}$ which in fact are derivatives of smooth functions.

Proposition 3.7. For every $k, m \in \mathbb{N}$, there exists a $C^{1}$ smooth Lipschitz function $f: \mathbb{R}^{k} \rightarrow \mathbb{R}^{m}$ so that $f$ vanishes outside a bounded set and the unit ball of $\mathscr{L}\left(\mathbb{R}^{k}, \mathbb{R}^{m}\right)$ is contained in $f^{\prime}\left(\mathbb{R}^{k}\right)$. In particular, for every $m \in \mathbb{N}$ there is a continuous path $g:[0,1] \rightarrow$ $\mathbb{R}^{m}$ whose image contains the unit ball of $\mathbb{R}^{m}$ and so that $g$ is the derivative of a $C^{1}$ smooth Lipschitz path $f:[0,1] \rightarrow \mathbb{R}^{m}$.

Proof of Theorem $3 \cdot 1$. It is clear that (2) implies (1). Let us see that (1) implies (2) too. Assume that dens $X=\operatorname{dens} \mathscr{L}_{s}^{m}(X ; Y)=\kappa$. Then it is easily seen that dens $X=$ dens $\mathscr{L}_{s}^{k}(X ; Y)=\kappa$ for all $k$ with $0 \leqslant k \leqslant m$. It is enough to see that for any $k$ with $0 \leqslant k \leqslant m$ there exists a $C^{p}$ smooth function $g: X \rightarrow Y$ with support on $B_{X}$ so that $g^{(k)}(X)$ contains the unit ball of $\mathscr{L}_{s}^{k}(X ; Y)$. Indeed, once this is shown, we can take a disjoint sequence of balls of the same diameter, $2 r$, contained in the unit ball $B_{X}$, say 
$B\left(z_{n}, r\right), n=0,1,2,3, \ldots$, and $C^{p}$ smooth functions $b_{0}, b_{1}, b_{2}, \ldots, b_{m}$, with support in $B_{X}$ and taking values in $Y$, so that $b_{k}^{(k)}(X)$ contains the unit ball of $\mathscr{L}_{s}^{k}(X ; Y)$ for every $k$; then the function $f: X \rightarrow Y$ defined by

$$
f(x)=\sum_{k=0}^{m} r^{k} b_{k}\left(\frac{x-z_{k}}{r}\right)
$$

is clearly a $C^{p}$ smooth bump with the property that $f^{(k)}(X)=b_{k}^{(k)}(X)$ contains the unit ball of $\mathscr{L}_{s}^{k}(X ; Y)$ for every $k$.

So let us prove that for a fixed $k$ with $0 \leqslant k \leqslant p$ there exists a $C^{p}$ smooth function $g: X \rightarrow Y$ with support on $B_{X}$ so that $g^{(k)}(X)$ contains the unit ball of $\mathscr{L}_{s}^{k}(X ; Y)$.

Since $X$ has a $C^{p}$ bump function with bounded derivatives, by composing it with a suitable real function, we can obtain a $C^{p}$ function $h: X \rightarrow[0,1]$ such that for some $M_{j}>0, j=1,2, \ldots$, and $M \geqslant 3$ we have $h(x)=1$ whenever $\|x\| \leqslant 2, h(x)=0$ if $\|x\| \geqslant M$, and $\left\|h^{(j)}\right\|_{\infty}=\sup _{x \in X}\left\|h^{(j)}(x)\right\| \leqslant M_{j}$.

Let us fix $\varepsilon$, where $0<2 M \varepsilon<\frac{1}{2}$, and select a $2 M \varepsilon$-separated collection of points $\left(z_{\alpha}\right)_{\alpha \in \Gamma}$ in $\frac{1}{2} B_{X}$ with $\operatorname{card}(\Gamma)=\kappa=$ dens $X$. The balls $B\left(z_{\alpha}, M \varepsilon\right), \alpha \in \Gamma$, are all disjoint and contained in $B=B_{X}$. As in [1], we define chains of balls

$$
U_{j}^{s}:=B_{\left(\alpha_{1}, \alpha_{2}, \ldots, \alpha_{j}\right)}=z_{\alpha_{1}}+\varepsilon z_{\alpha_{2}}+\cdots+\varepsilon^{j-1} z_{\alpha_{j}}+\varepsilon^{j} B
$$

for $s=\left(\alpha_{1}, \alpha_{2}, \ldots, \alpha_{j}, \ldots\right) \in \Gamma^{\mathbb{N}}$. There is a bijection between the chains of balls $\left(U_{j}^{s}\right)$ and the set of sequences $\Gamma^{\mathbb{N}}$; besides, the intersection of any chain of these balls consists exactly of the point $\bigcap_{j=1}^{\infty} B_{\left(\alpha_{1}, \alpha_{2}, \ldots, \alpha_{j}\right)}=\sum_{j=1}^{\infty} \varepsilon^{j-1} z_{\alpha_{j}}$.

Now, for a fixed $\varepsilon>0$ such that $\varepsilon<1 / 4 M$, since dens $\left(\mathscr{L}_{s}^{k}(X ; Y)\right)=\operatorname{card}(\Gamma)$, we can take a family $\left(Q_{\alpha}\right)_{\alpha \in \Gamma}$ which is dense in the unit ball of $\mathscr{L}_{s}^{k}(X ; Y)$, and a corresponding family $\left(P_{\alpha}\right)_{\alpha \in \Gamma}$ of $k$-homogeneous polynomials from $X$ into $Y$ so that $Q_{\alpha}$ is the $k$ th derivative of $P_{\alpha}$ for each $\alpha$. Notice that in the case $k=0$ we are dealing with a dense subset $\left(y_{\alpha}\right)_{\alpha \in \Gamma}$ of $Y$.

Next, for every $n \geqslant 1$ we can define $\delta_{n}=\varepsilon^{n^{2}-1}$, and

$$
g_{n}(x)=\sum_{\left(\alpha_{1}, \alpha_{2}, \ldots, \alpha_{n}\right) \in \Gamma^{n}} \delta_{n} h\left(\frac{x-\sum_{i=1}^{n} \varepsilon^{i-1} z_{\alpha_{i}}}{\varepsilon^{n}}\right) P_{\alpha_{n}}(x)
$$

for all $x \in X$. It is clear that $g_{n}$ is $C^{p}$ smooth with bounded derivatives, and its support is in $B$. Notice also that every $x \in X$ has a neighbourhood $V_{x}$ so that all but one of the terms in the sum defining $g_{n}(y)$ are zero for $y \in V_{x}$. Besides, we have that $g_{n}^{(k)}\left(B_{\left(\alpha_{1}, \alpha_{2}, \ldots, \alpha_{n}\right)}\right)=\delta_{n} Q_{\alpha_{n}}$ for all $\left(\alpha_{1}, \alpha_{2}, \ldots, \alpha_{n}\right) \in \Gamma^{n}$. Bearing in mind that the $i$ th derivative of $h$ is uniformly bounded by $M_{i}$, the construction of $g_{n}$, and the fact that if a $k$-homogeneous polynomial $P$ has its $k$ th derivative $Q$ bounded by 1 then all the derivatives of $P$ are bounded by 1 as well (this is an immediate inductive application of the mean value theorem), we can estimate the norm of the $j$ th derivative of $g_{n}$ as follows

$$
\left\|g_{n}^{(j)}(x)\right\| \leqslant \delta_{n} \sum_{i=1}^{j}\left(\begin{array}{c}
j \\
i
\end{array}\right) \frac{M_{i}}{\varepsilon^{n i}} \leqslant \delta_{n} \varepsilon^{-n j} M_{j} \sum_{i=1}^{j}\left(\begin{array}{c}
j \\
i
\end{array}\right) \leqslant 2^{j} M_{j} \varepsilon^{n(n-j)-1}
$$

for all $x \in X$ and $n \in \mathbb{N}$, and for every $j$ with $0 \leqslant j \leqslant p$.

Since the series $\sum_{n=1}^{\infty} 2^{j} M_{j} \varepsilon^{n(n-j)-1}$ are convergent for all $j=0,1,2, \ldots$, this implies that the series of derivatives $\sum_{n=1}^{\infty} g_{n}^{(j)}(x)$ converge uniformly on $X$ (for all 
$0 \leqslant j \leqslant p$ ), and therefore the function $g: X \rightarrow Y$ defined by

$$
g(x)=\sum_{n=1}^{\infty} g_{n}(x)
$$

is $C^{p}$ smooth with bounded derivatives, and $g^{(j)}(x)=\sum_{n=1}^{\infty} g_{n}^{(j)}(x)$.

Let us now see that $g^{(k)}(X)$ contains the unit ball of $\mathscr{L}_{s}^{k}(X ; Y)$. By the construction of the $g_{n}$ and $g$ it is clear that

$$
g^{(k)}\left(\partial B_{\left(\alpha_{1}, \ldots, \alpha_{k}\right)}\right)=Q_{\alpha_{1}}+\delta_{2} Q_{\alpha_{2}}+\cdots+\delta_{n} Q_{\alpha_{n}}
$$

for every chain of balls $\left(B_{\left(\alpha_{1}, \ldots, \alpha_{n}\right)}\right)_{n \in \mathbb{N}}$; then, for $x:=\bigcap_{n=1}^{\infty} B_{\left(\alpha_{1}, \alpha_{2}, \ldots, \alpha_{n}\right)}$, by the continuity of $g^{(k)}$ we get that $g^{(k)}(x)=\sum_{n=1}^{\infty} \delta_{n} Q_{\alpha_{n}}$. Since $\left(Q_{\alpha}\right)_{\alpha \in \Gamma}$ is dense in the unit ball of $\mathscr{L}_{s}^{k}(X ; Y)$ it is clear that every $Q$ in this ball can be written as a series $Q=\sum_{n=1}^{\infty} \delta_{n} Q_{\alpha_{n}}$ for some sequence $\left(\alpha_{n}\right) \in \Gamma^{\mathbb{N}}$, so we can conclude that $g^{(k)}(X)$ contains the unit ball of $\mathscr{L}_{s}^{k}(X ; Y)$.

Finally, in order to obtain a $C^{p}$ smooth surjection $b: X \rightarrow Y$ such that $b^{(k)}(X)=$ $\mathscr{L}_{s}^{k}(X ; Y)$ for every $k=0,1, \ldots, m$, we only have to take a sequence $\left(\alpha_{1}, \alpha_{2}, \ldots, \alpha_{n}, \ldots\right) \in \Gamma^{\mathbb{N}}$ with $\alpha_{i} \neq \alpha_{j}$ if $i \neq j$, a $C^{p}$ smooth function $f: X \rightarrow Y$ with support in $B_{X}$ and such that $f^{(k)}(X)$ contains the unit ball of $\mathscr{L}_{s}^{k}(X ; Y)$ for every $k=0,1,2, \ldots, m$, and put

$$
b(x)=\sum_{n=1}^{\infty} n f\left(\frac{x-z_{\alpha_{n}}}{\varepsilon}\right)
$$

for all $x \in X$.

Proof of Proposition 3.7. Let us denote $X=\mathbb{R}^{k}$ and $Y=\mathbb{R}^{m}$. Fix $0<\varepsilon<\frac{1}{8}$ and take a $\varepsilon$-net $\left(T_{\lambda}\right)_{\lambda \in \Gamma}$ in $B_{\mathscr{L}(X, Y)}$, i.e. to say, a maximal family in $B_{\mathscr{L}(X, Y)}$ satisfying $\left\|T_{\lambda}-T_{\lambda^{\prime}}\right\| \geqslant \varepsilon$, whenever $\lambda \neq \lambda^{\prime}$. Notice that $\Gamma$ is finite. Denote $\Sigma_{n}=\Gamma^{n}$ and $\Sigma^{\prime}=$ $\bigcup_{n} \Sigma_{n}$. Now, consider the sequence of positive real numbers $\left\{r_{n}\right\}_{n}$, where $r_{n+1}<r_{n} / 8$, as well as a family of points $\left(x_{\sigma}\right)_{\sigma \in \Sigma^{\prime}}$ in $\frac{1}{2} B_{X}$ satisfying:

(1) $B\left(x_{\sigma}, r_{n}\right) \subset \frac{1}{2} B_{X}$, whenever $|\sigma|=n$;

(2) if $\sigma \neq \sigma^{\prime}$ have length $n$, then $\left\|x_{\sigma}-x_{\sigma^{\prime}}\right\| \geqslant 2 r_{n}$;

(3) if $|\sigma|=n,\left|\sigma^{\prime}\right|=n+1$ and $\sigma<\sigma^{\prime}$, then $\left\|x_{\sigma}-x_{\sigma^{\prime}}\right\|=r_{n} / 4$.

Let us denote by $h$ a $C^{\infty}$ smooth bump on $X$ so that $h(x)=1$ for $\|x\| \leqslant \frac{1}{2}, h(x)=0$ whenever $\|x\| \geqslant 1$, and $h(X) \subset[0,1]$. Define

$$
f_{n}(x)=\sum_{\sigma \in \Sigma_{n}} \varepsilon^{n-1}\left\langle T_{\sigma(n)}, x-x_{\sigma}\right\rangle h\left(r_{n}^{-1}\left(x-x_{\sigma}\right)\right)
$$

and

$$
f(x)=\sum_{n} f_{n}(x) .
$$

Then $f$ is a $C^{1}$ smooth Lipschitz function from $X$ to $Y$ since $\sum_{n} f_{n}^{\prime}$ uniformly converges on $\mathbb{R}^{n}$. Also, notice that every $T \in B_{\mathscr{L}(X, Y)}$ can be written as a sum $\sum_{n} \varepsilon^{n-1} T_{\gamma(n)}$, for some $\gamma \in \Gamma^{\mathbb{N}}$, because $\left(T_{\lambda}\right)$ is a $\varepsilon$-net in $B_{\mathscr{L}(X, Y)}$. Thus, if $x=$ $\lim _{n} x_{\gamma_{n}}$, then $f^{\prime}(x)=\sum_{n} \varepsilon^{n-1} T_{\gamma(n)}$. This implies that $B_{\mathscr{L}(X, Y)} \subset f^{\prime}(X)$ and finishes the proof. 
4. Some applications: smooth approximation of starlike bodies by starlike bodies with many tangent hyperplanes

Before stating our results, let us recall the definition and a few properties of starlike bodies. A closed subset $A$ of a Banach space $X$ is said to be a starlike body provided there exists a point $x_{0} \in \operatorname{int} A$ such that each ray emanating from $x_{0}$ meets the boundary of $A$ at most once. In this case we will say that $A$ is starlike with respect to $x_{0}$. One can always assume that such bodies are starlike with respect to the origin (up to a suitable translation); so we can define the gauge or Minkowski functional of a starlike body $A$ as $\mu_{A}(x)=\inf \left\{\lambda>0 \mid \frac{1}{\lambda} x \in A\right\}$ for all $x \in X$. It is easily seen that for every starlike body $A$ its gauge $\mu_{A}$ is a continuous function which satisfies $\mu_{A}(r x)=r \mu_{A}(x)$ for every $r \geqslant 0$. Moreover, $A=\left\{x \in X \mid \mu_{A}(x) \leqslant 1\right\}$, and $\partial A=\left\{x \in X \mid \mu_{A}(x)=1\right\}$, where $\partial A$ stands for the boundary of $A$. Conversely, if $\psi: X \rightarrow[0, \infty)$ is continuous and satisfies $\psi(\lambda x)=\lambda \psi(x)$ for all $\lambda \geqslant 0$, then $A_{\psi}=\{x \in X \mid \psi(x) \leqslant 1\}$ is a starlike body. We will say that $A$ is a $C^{p}$ smooth starlike body provided its Minkowski functional $\mu_{A}$ is $C^{p}$ smooth on the set $X \backslash \mu_{A}^{-1}(0)$. This is equivalent to saying that $\partial A$ is a 1 -codimensional $C^{p}$ smooth submanifold of $X$ whose tangent hyperplanes do not contain any ray coming from the origin.

It is also worth noting that every Banach space having a $C^{p}$ smooth (Lipschitz) bump function has a $C^{p}$ smooth (Lipschitz) bounded starlike body too [7] (the converse is also clearly true).

Theorem 4·1. Let $X$ be an infinite-dimensional Banach space with a separable dual $X^{*}$. Then, for every bounded starlike body $A$ and every $\varepsilon>0$ there exists a $C^{1}$ smooth starlike body $D$ so that $\left|\mu_{D}(x)-\mu_{A}(x)\right| \leqslant \varepsilon$ for all $x$ with $\|x\| \leqslant 1$, and the cone of tangent hyperplanes to $D, \mathscr{C}(D)$, fills the dual space $X^{*}$.

If $X$ is separable but $X^{*}$ is not, the same conclusion holds replacing $C^{1}$ smoothness with Gâteaux smoothness.

The idea behind the proof of this result is similar to that of [1, theorem 1.2], but is slightly complicated by some difficulties peculiar to smooth approximation of starlike bodies. First we approximate our starlike body $A$ by a $C^{1}$ smooth starlike body $V$. Then we modify $V$ by creating a number of suitably located small flat patches on its boundary, and upon each of those patches we put a small $C^{1}$ smooth bump whose set of gradients is large enough. The starlike body $D$ thus constructed will have the required properties. For the reader's convenience we will split the most technical part of the proof into several lemmas.

Lemma $4 \cdot 2$. Let $X$ be a Banach space with separable dual $X^{*}$. For every bounded starlike body $A$ and for every $\varepsilon>0$ there exists a $C^{1}$ smooth starlike body $V=V_{\varepsilon}$ so that $\left|\mu_{A}(x)-\mu_{V}(x)\right| \leqslant \varepsilon$ for every $x \in B_{X}$.

Proof. Since $X$ has a separable dual, it has $C^{1}$ smooth partitions of unity, and a $C^{1}$ smooth equivalent norm $\|\cdot\|$ too (see [7]). Therefore, a given continuous function such as $\mu_{A}$ can be uniformly approximated by $C^{1}$ smooth functions on $X$; in particular there exists a $C^{1}$ smooth function $f: X \rightarrow \mathbb{R}$ so that $\left|\mu_{A}(x)-f(x)\right| \leqslant \varepsilon$ for all $x \in X$. It can be assumed that $\varepsilon$ is small enough so that $0<\varepsilon<\inf \left\{\mu_{A}(x):\|x\|=1\right\}$ 
and hence $\inf \{f(x):\|x\|=1\}>0$. Now define $\psi: X \rightarrow[0, \infty)$ by

$$
\psi(x)=\|x\| f\left(\frac{x}{\|x\|}\right)
$$

for all $x \in X \backslash\{0\}$, and $\psi(0)=0$. It is clear that $\psi$ is a positive-homogeneous continuous function which is $C^{1}$ smooth away from 0 , and therefore

$$
V=\{x \in X: \psi(x) \leqslant 1\}
$$

is a $C^{1}$ smooth starlike body whose Minkowski functional is $\mu_{V}=\psi$. Besides, we have that

$$
\left|\mu_{A}(x)-\mu_{V}(x)\right|=\left|\mu_{A}(x)-f(x)\right| \leqslant \varepsilon
$$

for all $x \in X$ with $\|x\|=1$ and, by the positive-homogeneity of $\mu_{A}$ and $\mu_{V}$, this implies that

$$
\left|\mu_{A}(x)-\mu_{V}(x)\right| \leqslant \varepsilon
$$

for all $x \in B_{X}$.

Lemma 4·3. Let $X$ be a Banach space and let $A$ be a $C^{1}$ smooth bounded starlike body in $X$. For every $z^{*} \in X^{*}$ and $z \in X$ so that $z^{*}(z)=\|z\|=\left\|z^{*}\right\|=1$, and for every $\varepsilon>0$, $\delta>0$, there exist a $C^{1}$ smooth starlike body $V=V_{z, \varepsilon}$ and $r \in(0, \delta)$ so that:

(1) $\mu_{V}(x)=\mu_{A}(x)$ for all $x$ with $\|x\|=1$ and $\|x-z\| \geqslant 2 r$; that $i s$, A and $V$ coincide outside the cone $\{\lambda x: \lambda \geqslant 0,\|x-z\|<2 r,\|x\|=1\}$;

(2) $\left|\mu_{V}(x)-\mu_{A}(x)\right| \leqslant \varepsilon$ for all $x \in B_{X}$;

(3) $\mu_{V}(x)=\mu_{A}(z) z^{*}(x)$ for all $x$ such that $\|x\|=1$ and $\|x-z\| \leqslant r$; that is, $\partial V$ has a flat patch (of radius $r / \mu_{A}(z)$ ) parallel to the hyperplane ker $z^{*}$ around the point $z^{\prime}=\left(1 / \mu_{A}(z)\right) z \in \partial A$.

Proof. Since $z^{*}$ and $\mu_{A}$ are continuous at $z$, and $z^{*}(z)=1$, there exists $r>0$ so that $\left.\left\|z^{*}(x)\right\| \geqslant 1 / 2, \mid \mu_{A}(z) z^{*}(x)-\mu_{A}(z)\right) \mid \leqslant \varepsilon / 2$, and $\left|\mu_{A}(x)-\mu_{A}(z)\right| \leqslant \varepsilon / 2$, whenever $\|x-z\| \leqslant 2 r$; of course we can assume that $r<\delta$. Let $\theta: \mathbb{R} \rightarrow[0,1]$ be a $C^{\infty}$ smooth function so that $\theta$ is non-decreasing, $\theta(t)=0$ for $t \leqslant r$, and $\theta(t)=1$ for $t \geqslant 2 r$. Let us define $\psi: X \rightarrow[0, \infty)$ by

$$
\psi(x)=\|x\|\left[\theta\left(\left\|\frac{x}{\|x\|}-z\right\|\right) \mu_{A}\left(\frac{x}{\|x\|}\right)+\left(1-\theta\left(\left\|\frac{x}{\|x\|}-z\right\|\right)\right) \mu_{A}(z) z^{*}\left(\frac{x}{\|x\|}\right)\right]
$$

for all $x \in X \backslash\{0\}$, and $\psi(0)=0$. It is clear that $\psi$ is $C^{1}$ smooth away from the origin and satisfies $\psi(\lambda x)=\lambda \psi(x)$ for all $\lambda \geqslant 0, x \in X$; therefore

$$
V=\{x \in X: \psi(x) \leqslant 1\}
$$

is a $C^{1}$ smooth starlike body whose gauge is $\mu_{V}=\psi$. From the definition of $\theta$ and $\psi$ it is obvious that $\psi=\mu_{V}$ fulfills conditions (1) and (3). Let us check that $\psi$ also satisfies (2); since $\psi$ and $\mu_{A}$ are both positive-homogeneous it is enough to do so for every $x$ on the unit sphere. If $\|x\|=1$ then

$$
\begin{aligned}
\left|\psi(x)-\mu_{A}(x)\right| & =(1-\theta(\|x-z\|))\left|\mu_{A}(z) z^{*}(x)-\mu_{A}(x)\right| \\
& \leqslant\left|\mu_{A}(z) z^{*}(x)-\mu_{A}(x)\right| \leqslant\left|\mu_{A}(z) z^{*}(x)-\mu_{A}(z)\right|+\left|\mu_{A}(z)-\mu_{A}(x)\right| \\
& \leqslant \frac{\varepsilon}{2}+\frac{\varepsilon}{2}=\varepsilon,
\end{aligned}
$$

so we have what we want. 
Lemma 4.4. Let $X$ be an infinite-dimensional Banach space and let $A$ be a $C^{1}$ smooth bounded starlike body in $X$. For every $z^{*} \in X^{*}$ and $z \in X$ so that $z^{*}(z)=\|z\|=\left\|z^{*}\right\|=$ 1 , consider the decomposition $X=H \oplus[z]=H \times \mathbb{R}$, where $H=\operatorname{Ker} z^{*}$. Then, for every $\varepsilon>0, \delta>0$, there exist a $C^{1}$ smooth starlike body $W=W_{z, \varepsilon}$ and $r \in(0, \delta)$ so that:

(1) A and $W$ coincide outside the half-cylinder $\{x=(h, t) \in X:\|h\| \leqslant r, t>0\}$;

(2) $\left|\mu_{W}(x)-\mu_{A}(x)\right| \leqslant \varepsilon$ whenever $\|x\| \leqslant 1$;

(3) for every hyperplane $F$ not containing any vector of the cone $\{x=(h, t) \in X:|t|>$ $2\|h\|\}$ there exists $y \in \partial W \cap\{x=(h, t) \in X: t>0,\|h\| \leqslant r\}$ such that $y+F$ is tangent to $\partial W$ at $y$.

Proof. From Theorem $3 \cdot 1$ we know that there exists a $C^{1}$ smooth Lipschitz bump $f: H \rightarrow \mathbb{R}$ with support in the unit ball of $H, B_{H}$, so that $f^{\prime}\left(B_{H}\right)$ contains the unit ball of the dual $H^{*}$, denoted by $B_{H^{*}}$. Let $M / 2$ be the Lipschitz constant of $f$. Since $f$ is $M / 2$-Lipschitz and has a bounded support, $f$ is bounded too. Hence, for $s>0$ small enough the bump function $f_{s}(h)=(s / 4) f(8 h / s)$ is $M$-Lipschitz, takes values in $[0, a]$, where

$$
a=\min \left\{\frac{\varepsilon}{16\left[\mu_{A}(z)^{2}+1\right]}, \frac{\mu_{A}(z)}{4}\right\},
$$

has its support in $B_{H}(0, s / 8)$, and has the property that $f_{s}^{\prime}\left(B_{H}(0, s / 8)\right)$ contains the ball $2 B_{H^{*}}$.

From this fact and from Lemma $4 \cdot 3$ we can take $r>0$ and a $C^{1}$ smooth starlike body $V$ so that the bump $f_{r}$ has the properties listed above,

$$
r<\min \left\{a, \frac{1}{M \mu_{A}(z)}\right\}
$$

and

(1) $\mu_{V}(x)=\mu_{A}(x)$ for all $x$ with $\|x\|=1$ and $\|x-z\| \geqslant r / 2$;

(2) $\left|\mu_{V}(x)-\mu_{A}(x)\right| \leqslant \varepsilon / 2$ for all $x \in B_{X}$;

(3) $\mu_{V}(x)=\mu_{A}(z) z^{*}(x)$ for all $x$ such that $\|x\|=1$ and $\|x-z\| \leqslant r / 4$.

Since $\{x \in X:\|x-z\| \leqslant r / 2,\|x\|=1, t>0\} \subseteq\{x=(h, t) \in X:\|x\|=1,\|h\| \leqslant r\}$, and $\{x=(h, t) \in X:\|x\|=1,\|h\| \leqslant r / 8, t>0\} \subseteq\{x \in X:\|x-z\| \leqslant r / 4,\|x\|=1\}$, this implies that

(1) $A$ and $V$ coincide outside the half-cylinder $\{x=(h, t) \in X:\|h\| \leqslant r, t>0\}$;

(2) $\left|\mu_{V}(x)-\mu_{A}(x)\right| \leqslant \varepsilon / 2$ for all $x \in B_{X}$;

(3) the boundary $\partial V$ and the hyperplane $\left\{x \in X: z^{*}(x)=\mu_{A}(z)\right\}$ have the same intersection with the cylinder $\{x=(h, t) \in X:\|h\| \leqslant r / 8\}$.

Now define

$$
W=\left\{x=(h, t) \in X: 0 \leqslant t \leqslant \frac{1}{\mu_{A}(z)}+f_{r}(h),\|h\| \leqslant \frac{r}{8}\right\} \cup\left\{x \in X: \mu_{V}(x) \leqslant 1\right\} .
$$

Let us see that $W$ satisfies the required properties. We first check that $W$ is a $C^{1}$ smooth starlike body. Let us take $y \in \partial W$ and see that the ray $\{\lambda y: \lambda \geqslant 0\}$ meets the boundary $\partial W$ only once, exactly at the point $y$. Write $y=\left(h_{y}, t_{y}\right) \in X$. Since the sets $V$ and $W$ coincide outside the half-cylinder $\{(h, t) \in X:\|h\| \leqslant r / 8, t>0\}$ and $V$ is starlike we only have to check the case $\left\|h_{y}\right\| \leqslant r / 8$ and $t_{y}>0$. We have

$$
\frac{t_{y}}{\left\|h_{y}\right\|} \geqslant \frac{1 / \mu_{A}(z)}{r / 8} \geqslant \frac{1}{r \mu_{A}(z)}>M
$$


If there were another point $y^{\prime}=\lambda y, \lambda \neq 1, \lambda>0$, such that $y^{\prime} \in \partial W$; then we would have

$$
\frac{t_{y^{\prime}}-t_{y}}{\left\|h_{y^{\prime}}\right\|-\left\|h_{y}\right\|}=\frac{(\lambda-1) t_{y}}{(\lambda-1)\left\|h_{y}\right\|}>M
$$

but in fact

$$
\begin{aligned}
\frac{t_{y^{\prime}}-t_{y}}{\left\|h_{y^{\prime}}\right\|-\left\|h_{y}\right\|} & =\frac{1 / \mu_{A}(z)+f_{r}\left(h_{y^{\prime}}\right)-\left(1 / \mu_{A}(z)+f_{r}\left(h_{y}\right)\right)}{\left\|h_{y^{\prime}}\right\|-\left\|h_{y}\right\|} \\
& =\frac{f_{r}\left(h_{y^{\prime}}\right)-f_{r}\left(h_{y}\right)}{(\lambda-1)\left\|h_{y}\right\|}=\frac{\left|f_{r}\left(h_{y^{\prime}}\right)-f_{r}\left(h_{y}\right)\right|}{\left\|h_{y^{\prime}}-h_{y}\right\|} \leqslant M
\end{aligned}
$$

because $f_{r}$ is $M$-Lipschitz, a contradiction. Therefore $W$ is a bounded starlike body, and it is $C^{1}$ smooth because it is locally the graph of a $C^{1}$ smooth function whose tangent hyperplanes do not contain any ray emanating from the origin (this property is again guaranteed by the fact that $f_{r}$ is $M$-Lipschitz).

From the definition of $W$ it is obvious that $W$ satisfies property (1) of the statement. To check property (2), take $x=(h, t) \in X$ with $\|x\|=1, \lambda>0$, and assume that $(1 / \lambda) x \in \partial W \backslash \partial V$ (i.e. $\lambda=\mu_{W}(x) \neq \mu_{V}(x)$; the case $\mu_{W}(x)=\mu_{V}(x)$ is trivial). Then we have that $(1 / \lambda)\|h\| \leqslant r / 8$, and $(1 / \lambda) t=f_{r}((1 / \lambda) h)+\left(1 / \mu_{A}\right)(z)$, so that

$$
\begin{aligned}
\left|\frac{1}{\lambda}-\frac{1}{\mu_{A}(z)}\right| & \leqslant\left|\frac{1}{\lambda}-\frac{t}{\lambda}\right|+\left|\frac{t}{\lambda}-\frac{1}{\mu_{A}(z)}\right|=\frac{1-t}{\lambda}+\left|f_{r}\left(\frac{h}{\lambda}\right)\right| \\
& \leqslant \frac{1}{\lambda}\|h\|+a \leqslant \frac{r}{8}+a \leqslant 2 a,
\end{aligned}
$$

and therefore

$$
\left|\lambda-\mu_{A}(z)\right|=\left|\frac{\lambda-\mu_{A}(z)}{\lambda \mu_{A}(z)}\right| \leqslant \frac{4}{3} \mu_{A}(z)^{2}\left|\frac{1}{\lambda}-\frac{1}{\mu_{A}(z)}\right| \leqslant \frac{8 a}{3} \mu_{A}(z)^{2} \leqslant \frac{\varepsilon}{2},
$$

that is,

$$
\left|\mu_{W}(x)-\mu_{V}(x)\right|=\left|\lambda-\mu_{A}(z)\right| \leqslant \frac{\varepsilon}{2} .
$$

By combining this inequality with property (2) of $\mu_{V}$ we get that

$$
\left|\mu_{W}(x)-\mu_{A}(x)\right| \leqslant \varepsilon,
$$

and this holds for all $x$ with $\|x\|=1$.

Finally, let us check (3). Bearing in mind the construction of $W$, this amounts to seeing that $\left\{T \in X^{*} \mid T(h+t z) \neq 0\right.$ for all $h+t z \in X$ with $\left.|t|>2\|h\|\right\}$ is contained in the set $\left\{T \in X^{*} \mid \operatorname{Ker} T\right.$ is tangent to the graph of $\left.t=f_{r}(h)\right\}$. Let us check this inclusion.

Take $T \in X^{*}$ such that $T(h+t z) \neq 0$ for all $h+t z \in X$ with $|t|>2\|h\|$. Then $T(h) / T(z) \leqslant 2$ for all $h \in H$ with $\|h\| \leqslant 1$ (indeed, either $T(z)>0$ or $T(z)<0$; suppose for instance $T(z)>0$; then, for $\|h\|<1=-t / 2$ we have $T(h-2 z) \neq 0$ and $T(-2 z)<0$; since the set $\{h \in H:\|h\|<1\}$ is connected and $T$ is continuous this implies that $T(h)-2 T(z)=T(h-2 z)<0$ and therefore $T(h) / T(z) \leqslant 2$ for all $h \in H$ with $\|h\|<1)$. If we define $S \in H^{*}$ by $S(h)=T(h) / T(z)$ for all $h \in H$ this means that $\|S\|_{H^{*}} \leqslant 2$. Now, since $2 B_{H^{*}} \subseteq f_{r}^{\prime}\left(B_{H}(0, r / 8)\right)$, there must be some $h_{0} \in B_{H}(0, r / 8)$ such that $f_{r}^{\prime}\left(h_{0}\right)=-S$. Then we have that $T(h+t z)=T(h)+t T(z)=T(z)[S(h)+t]=$ 
$T(z)\left[-f_{r}^{\prime}\left(h_{0}\right)(h)+t\right]$, and since $T(z) \neq 0$ this means that $T(h+t z)=0$ if and only if $t=f_{r}^{\prime}\left(h_{0}\right)(h)$, that is, $\operatorname{Ker} T$ is tangent to the graph of $t=f_{r}(h)$ at the point $h_{0}+f_{r}\left(h_{0}\right) z$.

Proof of Theorem 4.1. Let $A$ be a bounded starlike body in $X$. For a given $\varepsilon>0$, $\varepsilon \leqslant \frac{1}{8}$, we have to find a $C^{1}$ smooth starlike body $D=D_{\varepsilon}$ so that the cone of its tangent hyperplanes, $\mathscr{C}(D)$, fills the dual space $X^{*}$, and

$$
\left|\mu_{D}(x)-\mu_{A}(x)\right| \leqslant \varepsilon
$$

for every $x \in B_{X}$. Thanks to Lemma $4 \cdot 2$, we can assume that $A$ is $C^{1}$ smooth. Let $\left\{z_{\alpha}\right\}_{\alpha \in I}$ be a $\varepsilon$-net on the unit sphere $S_{X}$. For every $\alpha \in I$ (by the Hahn-Banach theorem) we can choose a $z_{\alpha}^{*} \in X^{*}$ so that $z_{\alpha}^{*}\left(z_{\alpha}\right)=1=\left\|z_{\alpha}^{*}\right\|$. Let us denote $H_{\alpha}=\operatorname{Ker} z_{\alpha}^{*}$. Now, for every $\alpha \in I$, by Lemma $4 \cdot 4$, we can take $r_{\alpha}>0$ and a $C^{1}$ smooth starlike body $W_{\alpha}$ so that:

(1) $A$ and $W_{\alpha}$ coincide outside the half-cylinder $\left\{x=(h, t) \in X=H_{\alpha} \oplus\left[z_{\alpha}\right]:\|h\| \leqslant\right.$ $\left.r_{\alpha}, t>0\right\}$

(2) $\left|\mu_{W_{\alpha}}(x)-\mu_{A}(x)\right| \leqslant \varepsilon$ whenever $\|x\| \leqslant 1$;

(3) for every hyperplane $F$ not containing any vector of the cone $\left\{(h, t) \in H_{\alpha} \oplus\right.$ $\left.\left[z_{\alpha}\right]:|t|>2\|h\|\right\}$ there exists $y \in \partial W_{\alpha} \cap\left\{(h, t) \in H_{\alpha} \oplus\left[z_{\alpha}\right]: t>0,\|h\| \leqslant r_{\alpha}\right\}$ such that $y+F$ is tangent to $\partial W_{\alpha}$ at $y$;

moreover, the $r_{\alpha}$ can be chosen small enough so that the sets

$$
\partial W_{\alpha} \cap\left\{(h, t) \in H_{\alpha} \oplus\left[z_{\alpha}\right]:\|h\| \leqslant r_{\alpha}, t>0\right\}
$$

are pairwise disjoint. For each $\alpha \in I$, let us denote the gauge of $W_{\alpha}$ by $\mu_{\alpha}$.

Now consider the union of all these bodies,

$$
D=\bigcup_{\alpha \in I} W_{\alpha} .
$$

Let us see that $D$ is a bounded $C^{1}$ smooth starlike body. Define $\psi: X \rightarrow(0, \infty)$ by

$$
\psi(x)=\inf _{\alpha \in I} \mu_{\alpha}(x) .
$$

It is obvious that $\psi$ is positive homogeneous, and it is not difficult to check that for every $z \in S_{X}$ there exists some $\delta>0$ and some $\alpha \in I$ such that $\psi(x)=\mu_{\alpha}(x)$ for all $x \in S_{X}$ with $\|x-z\|<\delta$; since every functional $\mu_{\alpha}$ is $C^{1}$ smooth away from the origin, this implies that $\psi$ is $C^{1}$ smooth in $X \backslash\{0\}$. Therefore $\{x \in X \mid \psi(x) \leqslant 1\}$ is a $C^{1}$ smooth starlike body, and it is easily checked that $D=\{x \in X \mid \psi(x) \leqslant 1\}$, so that $\psi$ is the Minkowski functional of $D$. The fact that $\psi$ is locally some of the $\mu_{\alpha}$ also implies that for every $x \in S_{X}$ there is some $\alpha \in I$ so that

$$
\left|\psi(x)-\mu_{A}(x)\right|=\left|\mu_{\alpha}(x)-\mu_{A}(x)\right| \leqslant \varepsilon,
$$

which shows that $D$ approximates $A$ as it is required.

It only remains to prove that for every hyperplane $F$ of $X$ there is some $y \in \partial D$ such that $y+F$ is tangent to $\partial D$ at $y$. Since for each $\alpha$ the bodies $W_{\alpha}$ and $D$ are the same inside the half-cylinder $C_{\alpha}=\left\{h+t z_{\alpha} \in H_{\alpha} \oplus\left[z_{\alpha}\right]:\|h\| \leqslant r_{\alpha}, t>0\right\}$, all the hyperplanes of $X$ not containing any vector of $\left\{h+t z_{\alpha} \in H_{\alpha} \oplus\left[z_{\alpha}\right]:|t|>2\|h\|\right\}$ are tangent to $\partial W_{\alpha}$, and therefore tangent to $\partial A$ too, at some point of $\partial W_{\alpha} \cap C_{\alpha}=$ 
$\partial D \cap C_{\alpha}$. This means that the set

$$
\bigcup_{\alpha \in I}\left\{T \in X^{*} \mid T\left(h+t z_{\alpha}\right) \neq 0 \quad \text { for all } h+t z_{\alpha} \in H_{\alpha} \oplus\left[z_{\alpha}\right] \quad \text { with } \quad|t|>2\|h\|\right\}
$$

is contained in

$$
\left\{T \in X^{*} \mid y+\operatorname{Ker} T \text { is tangent to } \partial D \text { at some point } y \in \partial D\right\} .
$$

Therefore, in order to conclude the proof we only have to check that

$X^{*} \backslash\{0\}=\bigcup_{\alpha \in I}\left\{T \in X^{*} \mid T\left(h+t z_{\alpha}\right) \neq 0 \quad\right.$ for all $h+t z_{\alpha} \in H_{\alpha} \oplus\left[z_{\alpha}\right] \quad$ with $\left.\quad|t|>2\|h\|\right\}$.

Consider any $T \in X^{*}, T \neq 0$; we may assume $\|T\|=1$. Choose $z \in X,\|z\|=1$, such that $T(z)>1-\varepsilon$, and take $z_{\alpha}$ such that $\left\|z-z_{\alpha}\right\| \leqslant \varepsilon$. We have that $\left|T\left(z_{\alpha}\right)-T(z)\right| \leqslant$ $\left\|z-z_{\alpha}\right\| \leqslant \varepsilon$ and hence $T\left(z_{\alpha}\right) \geqslant T(z)-\varepsilon>1-2 \varepsilon>0$. Then, for every $h+t z_{\alpha} \in$ $H_{\alpha} \oplus\left[z_{\alpha}\right]$ with $t>2\|h\|>0$ we get

$$
\begin{aligned}
T\left(h+t z_{\alpha}\right) & =T(h)+t T\left(z_{\alpha}\right)>T(h)+t(1-2 \varepsilon) \geqslant-\|h\|+t(1-2 \varepsilon) \\
& >-\|h\|+2\|h\|(1-2 \varepsilon)=(1-4 \varepsilon)\|h\|>0 ;
\end{aligned}
$$

and in a similar way one checks that $T\left(h+t z_{\alpha}\right)<0$ for all $h+t z_{\alpha} \in H_{\alpha} \oplus\left[z_{\alpha}\right]$ with $t<-2\|h\|<0$. Therefore $T\left(h+t z_{\alpha}\right) \neq 0$ for all $h+t z_{\alpha} \in H_{\alpha} \oplus\left[z_{\alpha}\right]$ with $|t|>2\|h\|$. This concludes the proof of Theorem $4 \cdot 1$ in the $C^{1}$ smooth case.

Finally, let us say a few words about the Gâteaux smooth case. It is known that every separable Banach space $X$ has Gâteaux smooth partitions of the unity (see [7]), so both the statement and the proof of Lemma $4 \cdot 2$ remain valid in this case. The same applies to Lemma 4.3. A Gâteaux smooth norm can be used instead of a $C^{1}$ smooth norm when $X^{*}$ is not separable in those proofs. In the proofs of Lemma $4 \cdot 4$ and Theorem $4 \cdot 1$, we make implicit use of the implicit function theorem, which is not true for Gâteaux smooth functions; this is the only delicate point of the proof in the Gâteaux case. The techniques used herein can nonetheless be adapted with some care to avoid this problem. For instance, when showing that the Minkowski functional of a starlike body which is locally the graph of a uniformly Gâteaux smooth function is Gâteaux smooth too, one can consider the sections of the body with two-dimensional subspaces; these sections are then two-dimensional starlike bodies which are locally graphs of uniformly Gâteaux smooth functions of one real variable; since Gâteaux and Fréchet notions of differentiability coincide for functions of one real variable and uniformly Gâteaux functions have continuous derivatives, uniformly Gâteaux smoothness imply $C^{1}$ smoothness in this case, so the implicit function theorem can be used to ensure the Gâteaux smoothness of our two-dimensional starlike bodies, and since the tangent lines to those sections all belong to the same hyperplane, this shows that the original infinite-dimensional starlike body is Gâteaux smooth as well.

Remark $4 \cdot 5$. A careful examination of the proof of Theorem $4 \cdot 1$ reveals that this result is true and the same proof holds if only $X$ has smooth partitions of unity. Indeed, whenever we make use of a smooth norm, one could use the gauge of a $C^{p}$ smooth bounded starlike body instead. So we have the following result.

Theorem 4.6. Let $X$ be an infinite-dimensional Banach space with $C^{p}$ smooth partitions of unity. Then, for every bounded starlike body $A$ and every $\varepsilon>0$ there exists a 
$C^{p}$ smooth starlike body $D$ so that $\left|\mu_{D}(x)-\mu_{A}(x)\right| \leqslant \varepsilon$ for all $x$ with $\|x\| \leqslant 1$, and the cone of tangent hyperplanes to $D, \mathscr{C}(D)$, fills the dual space $X^{*}$.

Before finishing this section let us consider the much easier related question about smooth approximation of continuous functions by smooth functions whose derivatives are surjections. In the following proposition, the term 'smooth' means either $C^{p}$ smooth or Gâteaux smooth.

Proposition 4.7. Let $X$ be an infinite-dimensional Banach space. The following are equivalent:

(1) $X$ has smooth partitions of unity;

(2) every continuous function $g: X \rightarrow \mathbb{R}$ can be uniformly approximated by smooth functions $f: X \rightarrow \mathbb{R}$ with the property that $f^{\prime}(X)=X^{*}$.

Proof. It is clear (see [7, chapter 8]) that (2) implies (1). Let us see that (1) implies (2). For a given $\varepsilon>0$ and a continuous function $g: X \rightarrow \mathbb{R}$, take $\delta>0$ so that $|g(x)-g(0)| \leqslant \varepsilon / 4$ whenever $\|x\| \leqslant 2 \delta$. Since $X$ has smooth partitions of unity there exists a smooth function $h$ on $X$ so that $|g(x)-h(x)| \leqslant \varepsilon / 2$ for all $x \in X$, and $h(x)=g(0)$ whenever $\|x\| \leqslant \delta$. From Theorem $3 \cdot 1$ we know that there exists a smooth Lipschitz bump $F: X \rightarrow \mathbb{R}$, with support in $B_{X}$, so that $F^{\prime}\left(B_{X}\right)$ contains the unit ball of the dual $X^{*}$. Since $F$ is Lipschitz and has a bounded support, $F$ is itself bounded; we can assume that $F$ takes values in $[0,1]$. Pick $r$ so that $0<r<\delta / 4<1$, and a sequence $\left(z_{n}\right)$ of points in the ball $B(0, \delta / 2)$ so that $\left\|z_{n}-z_{m}\right\| \geqslant r$ for $n \neq m$. The bump function

$$
b(x)=\sum_{n=1}^{\infty} \frac{\varepsilon}{2} F\left(\frac{x-z_{n}}{r^{n}}\right)
$$

takes values in $[0, \varepsilon / 2]$, has its support in $B(0, \delta)$, and has the property that $b^{\prime}(X)=$ $X^{*}$. Now define

$$
f(x)=h(x)+b(x)
$$

for all $x \in X$. It is clear that $f^{\prime}(X)=b^{\prime}(X)=X^{*}$ and

$$
|f(x)-g(x)| \leqslant|f(x)-h(x)|+|h(x)-g(x)|=|b(x)|+|h(x)-g(x)| \leqslant \frac{\varepsilon}{2}+\frac{\varepsilon}{2}=\varepsilon
$$

for all $x \in X$.

Acknowledgements. This research started during a visit of M. J-.S. to the Laboratoire de Mathématiques Pures de Bordeaux. This author is indebted to the members of the Laboratoire for their kind hospitality.

\section{REFERENCES}

[1] D. Azagra and R. Deville. James' theorem fails for starlike bodies. J. Funct. Anal. 180 (2001), 328-346.

[2] D. Azagra and M. Jiménez-Sevilla. The failure of Rolle's theorem in infinite dimensional Banach spaces. J. Funct. Anal. 182 (2001), 207-226.

[3] D. Azagra and M. Jiménez-Sevilla. On the size of the sets of gradients of bump functions and starlike bodies on the Hilbert space. Preprint.

[4] S. M. Bates. On smooth non-linear surjections of Banach spaces. Israel. J. Math. 100 (1997), 209-220.

[5] J. M. Borwein, M. Fabian, I. Kortezov and P. D. Loewen. The range of the gradient of a continuously differentiable bump. J. Nonlinear Convex Anal. 2 (2001), 1-19. 
[6] G. Debs, G. Godefroy and J. Saint Raymond. Topological properties of the set of normattaining linear functionals. Canad. J. Math. 47 (2) (1995), 318-329.

[7] R. Deville, G. Godefroy and V. Zizler. Smoothness and renormings in Banach spaces. Pitman monograph and surveys in pure and applied mathematics, 64 (Longman Scientific \& Technical, Harlow, 1993).

[8] V. Dimant and I. Zaldouendo. Bases in spaces of multilinear forms on Banach spaces. J. Math. Anal. Appl. 200 (1996), 548-566.

[9] T. Dobrowolski. Weak bump functions and applications. Bull. Polish Acad. Sci. Math. 49 (4) (2001), 337-347.

[10] R. Gonzalo and J. A. Jaramillo. Compact polynomials between Banach spaces. Proc. Roy. Ir. Acad. 95A (2) (1995), 213-226.

[11] P. HÁ.JeK. Smooth functions on $c_{0}$. Israel J. Math. 104 (1998), 17-27.

[12] M. Jiménez-Sevilla and J. P. Moreno. A note on norm attaining functionals. Proc. Amer. Math. Soc. 126 (3) (1998), 1989-1997.

[13] R. P. Maleev and S. L. Troyanski. Smooth norms in Orlicz spaces. Canad. Math. Bull. 34 (1991), 74-82.

[14] R. R. Phelps. Convex functions, monotone operators and differentiability, 2nd edn. Lecture Notes in Mathematics, vol. 1364 (1993). 